\title{
Neurovascular Organotypic Culture Models Using Induced Pluripotent Stem Cells to Assess Adverse Chemical Exposure Outcomes
}

\author{
Eric H. Nguyen, ${ }^{1,2}$ Micah J. Dombroe, ${ }^{3}$ Debra L. Fisk, ${ }^{1,2}$ William T. Daly, ${ }^{1,4,5}$ \\ Christine M. Sorenson, ${ }^{6}$ William L. Murphy, ${ }^{1,4,5}$ and Nader Sheibani ${ }^{1,2,4,7}$
}

\begin{abstract}
Introduction: Human-induced pluripotent stem cells (iPSCs) represent a promising cell source for the construction of organotypic culture models for chemical toxicity screening and characterization.

Materials and Methods: To characterize the effects of chemical exposure on the human neurovasculature, we constructed neurovascular unit (NVU) models consisting of endothelial cells (ECs) and astrocytes (ACs) derived from human-iPSCs, as well as human brain-derived pericytes (PCs). The cells were cocultured on synthetic poly(ethylene glycol) (PEG) hydrogels that guided the self-assembly of capillary-like vascular networks. High-content epifluorescence microscopy evaluated dose-dependent changes to multiple aspects of NVU morphology. Results: Cultured vascular networks underwent quantifiable morphological changes when incubated with vascular disrupting chemicals. The activity of predicted vascular disrupting chemicals from a panel of 38 compounds (U.S. Environmental Protection Agency) was ranked based on morphological features detected in the NVU model. In addition, unique morphological neurovascular disruption signatures were detected per chemical. A comparison of PEG-based NVU and Matrigel $^{\mathrm{TM}}$-based NVU models found greater sensitivity and consistency in chemical detection by the PEG-based NVU models.

Discussion: We suspect that specific morphological changes may be used for discerning adverse outcome pathways initiated by chemical exposure and rapid mechanistic characterization of chemical exposure to neurovascular function.

Conclusion: The use of human stem cell-derived vascular tissue and PEG hydrogels in the construction of NVU models leads to rapid detection of adverse chemical effects on neurovascular stability. The use of multiple cell types in coculture elucidates potential mechanisms of action by chemicals applied to the model.
\end{abstract}

Keywords: acute toxicity, cardiotoxicity, high throughput, neurotoxicity, ocular toxicity, stem cells

\section{Introduction}

$\mathbf{T}$ HERE ARE CURRENTLY ESTIMATED $>68,000$ commercially used chemicals that have not been appropriately characterized for their adverse effects on human tissue function. The costs of testing these chemicals are projected to be $\$ 14$ billion and 54 million animal lives. ${ }^{1}$ Current methods for measuring the adverse effects of chemical exposure on human tissue function are currently limited to animal models or cell monoculture assays. These assays are either low throughput or lacking in physiological relevance that would successfully inform regulatory bodies of safe exposure limits to these chemicals.

Recently, in vitro organotypic culture models (OCMs) have been developed to simulate adverse chemical effects on tissue and organ function. ${ }^{2,3}$ OCMs are being constructed from increasingly advanced materials and human stem cell sources, including embryonic stem cells and induced

\footnotetext{
${ }^{1}$ Human Models for Analysis of Pathways Center, University of Wisconsin School of Medicine and Public Health, Madison, Wisconsin.

${ }^{2}$ Department of Ophthalmology and Visual Sciences, University of Wisconsin School of Medicine and Public Health, Madison, Wisconsin.

${ }^{3}$ School of Pharmacy, University of Wisconsin-Madison, Madison, Wisconsin.

Departments of ${ }^{4}$ Biomedical Engineering, ${ }^{5}$ Orthopedics and Rehabilitation, ${ }^{6}$ Pediatrics, and ${ }^{7}$ Cell and Regenerative Biology, University of Wisconsin School of Medicine and Public Health, Madison, Wisconsin.
} 
pluripotent stem cells (iPSCs). ${ }^{4} \mathrm{~A}$ critical tissue of interest for modeling chemical toxicity is the neurovasculature of the central nervous system (CNS), which supplies blood to the brain, spinal cord, and the eye.

Endothelial cells (ECs) of the neurovascular interact with supporting cell types, including astrocytes (ACs), pericytes (PCs), and neurons to form the neurovascular unit (NVU), ${ }^{4}$ which maintains stable blood flow to and from the CNS. Hypoxia, ischemia, and cerebral hypoperfusion resulting from NVU failure can cause the onset of neurodevelopmental or neurodegenerative diseases, including Alzheimer's disease, ${ }^{5-8}$ Parkinson's disease, ${ }^{9,10}$ autism spectrum disorders, ${ }^{11,12}$ and diabetic retinopathy. ${ }^{5-8,13,14}$ The connection between NVU failure and these diseases is often attributed to decreased blood supply to neurons, inflammation, oxidative stress, as well as the buildup of $\alpha \beta$-amyloid peptides and immunocytokines in the CNS., 8 ,15,16

Although there is currently a large body of information about chemicals that are known to cause neurodegeneration, less is known about chemicals that can disrupt the neurovasculature itself. Particularly, chemicals can disrupt not only EC function, but also the functions of ACs and PCs that regulate ECs and neurovascular functionality and stability. ACs and PCs work to regulate extracellular matrix (ECM) deposition in the neurovascular environment,${ }^{17}$ integrin and tight junction expression by ECs,${ }^{17}$ EC polarization, ${ }^{18}$ as well as regulation of molecular transporter protein expression and localization in EC membranes. ${ }^{17,19,20}$ To understand the risks that chemicals pose to neurovascular function and develop countermeasures against the effects of exposure, the mechanisms of action by disruptive chemicals on ECs, ACs, and PCs, and likely neurons, must be better understood.

A common assay of vascular tissue function is the tubulogenesis assay, which measures changes to the formation of branched capillary-like networks by ECs in response to external stimuli. ${ }^{21}$ Standard tubulogenesis assays make use of naturally derived ECM such as Matrigel ${ }^{\mathrm{TM}}$, collagen, and hyaluronic acid to induce the formation of networks by ECs. However, these materials are associated with batchto-batch variation and the presence of extraneous biological signals that potentially mask the effects of drug and chemical exposure. ${ }^{22-25}$ These limitations often result in decreased sensitivity and reproducibility of tubulogenesis assays.

Synthetic biomaterials, including poly(ethylene glycol) (PEG) hydrogels, ${ }^{26}$ may be preferred as cell culture substrates as their bioinert properties minimize the presence of extraneous biological signaling. ${ }^{26-29}$ We have previously developed PEG hydrogels that promote capillary network formation by ECs derived from iPSCs, ${ }^{30-33}$ allow vascular and neural integration in three dimensional organoids, ${ }^{34,35}$ and demonstrated superior sensitivity and repeatability of PEG-based tubulogenesis assays compared with equivalent Matrigel-based assays.

Pluripotent stem cells represent an abundant cell source for the construction of human tissue models. iPSC-derived ECs have the ability to form capillary-like networks in tissue culture systems. ${ }^{31-33,36}$ However, it is known that ECs display differential gene expression patterns based on their tissues of origin, and there is an emerging need to construct tissue-specific vasculature in OCMs. ${ }^{37}$ ECs with blood brain barrier functionality have been derived from pluripotent stem cells, ${ }^{38-40}$ and others were able to coculture iPSC- derived ECs with glioma cells to specify the ECs toward a neurovascular phenotype. ${ }^{41}$ This presents an opportunity to adapt iPSC-derived ECs for use in neurovascular OCMs. Similarly, ACs have been derived from iPSCs and provide a plentiful source of cells for the construction of NVU models. ${ }^{42-44}$

In this study, we generated an NVU consisting of iPSCderived ECs, iPSC-derived ACs, and human brain-derived PCs seeded, in direct contact, onto proangiogenic PEG hydrogels, and investigated adverse effects of chemical exposure on neurovascular vessel integrity. Our model quantifies compromised capillary network formation and stability as rapid readouts of chemically mediated NVU failure.

\section{Materials and Methods}

\section{$E C, P C$, and $A C$ culture and maintenance}

iPSC-derived ECs were purchased from Cellular Dynamics International (CDI, Madison, WI). Vials were expanded and banked upon receipt for use over the duration of these studies. Cells were cultured in complete i-Cell ${ }^{\circledR} \mathrm{EC}$ maintenance medium (CDI) according to the i-Cell EC maintenance protocol. Tissue culture-treated polystyrene T-75 culture flasks were coated with $30 \mu \mathrm{g} / \mathrm{mL}$ human plasma fibronectin (Corning, Corning, NY) for at least 30 minutes before use. Maintenance medium was changed every other day and cells were passaged every 4-5 days. Cells were retrieved using $0.25 \%$ trypsin/EDTA $1 \times$ solution (Thermo Fisher, Waltham, MA) and recovered in Vasculife ${ }^{\circledR}$ basal medium (Lifeline Technologies, Frederick, MD) supplemented with $10 \%$ fetal bovine serum (FBS). The cells were maintained in a humidified $37^{\circ} \mathrm{C}$ incubator with $5 \% \mathrm{CO}_{2}$ and used between 5 and 16 population doublings in all experiments.

To our knowledge, there are currently no commercially prepared iPSC-derived PCs available. This is hampered by the heterogeneous characteristic of vascular PCs in the same tissue, as well as in vascular beds of different tissues, and lack of specific markers. ${ }^{45}$ In this study, human fetal brain-derived PCs were purchased from ScienCell Laboratories (Carlsbad, CA). Cells were expanded and banked upon receipt for use over the duration of these studies. Cells were cultured in complete PC medium (ScienCell Laboratories). Tissue culture-treated polystyrene T-75 culture flasks were coated with $0.15 \%$ poly(Llysine) (PLL; molecular weight 70,000-150,000 Da; Millipore Sigma) in sterile $\mathrm{H}_{2} \mathrm{O}$ for at least 30 minutes before use. PC medium was changed every other day and cells were passaged every 4-5 days. Cells were retrieved using $0.25 \%$ trypsin/EDTA $1 \times$ solution (Thermo Fisher) and recovered in PC medium. The cells were maintained in a humidified $37^{\circ} \mathrm{C}$ incubator with $5 \% \mathrm{CO}_{2}$ and used between 5 and 16 population doublings in all experiments.

iPSC-derived ACs were purchased from CDI. Cells were expanded and banked upon receipt for use over the duration of these studies. Cells were cultured in AC growth medium consisting of low-glucose Dulbecco's modified Eagle medium (DMEM; Sigma) supplemented with 10\% FBS and $1 \times$ N2-supplement (Thermo Fisher). Tissue culture-treated polystyrene six-well plates were coated with $0.083 \mathrm{mg} / \mathrm{cm}^{2}$ Matrigel (Corning) for at least 30 minutes before use. AC growth medium was changed every other day and cells were passaged every 7-14 days. Cells were retrieved using $0.25 \%$ trypsin/EDTA $1 \times$ solution (Thermo Fisher) and recovered in DMEM supplemented with 10\% FBS. The cells 
were maintained in a humidified $37^{\circ} \mathrm{C}$ incubator with $5 \%$ $\mathrm{CO}_{2}$ and used between 5 and 16 population doublings in all experiments.

\section{Labeling cells with Cell Tracker $^{\mathrm{TM}}$ dye}

Cells to be included in NVU models were labeled using Cell Tracker fluorescent dyes (Thermo Fisher) 24 hours before seeding. In brief, ECs or PCs in T-75 flasks were rinsed in DMEM for 5 minutes and stained with $10 \mathrm{~mL}$ of either 1.3 $\mu \mathrm{M}$ Cell Tracker Red CMPTX, 1.0 $\mu \mathrm{M}$ Cell Tracker Green CMFDA, or $10 \mu \mathrm{M}$ Cell Tracker Blue CMF2HC in DMEM for 45 minutes. ACs in six-well plates were similarly stained using $3 \mathrm{~mL}$ staining solution per well using identical concentrations of Cell Tracker dye. Afterward, the cells were rinsed again in DMEM for 5 minutes before incubating in their separate cell-specific growth medium overnight to allow the cells to sufficiently recover from the staining process.

\section{Determination of culture conditions \\ for NVU tubulogenesis assays}

Six chemically defined hydrogel conditions were examined in a hydrogel array format (Fig. 1) for their ability to induce capillary-like network formation by ECs (Fig. 2A). Refer to Supplementary Methods for information on generating hydrogel formulations and constructing hydrogel arrays. Eight experimental replicates were performed per condition, but deformed or nonlevel hydrogels were excluded from analysis. Norbornene-functionalized PEG (PEGNB) molecule concentrations varied between 2.5 and $3.5 \mathrm{mM}$ to control hydrogel modulus. Head-to-tail cyclized Arg-Gly-Asp[d-Phe]-Cys (cyclic RGD; Genscript, Piscataway, NJ) and Cys-Arg-Gly-Asp-Ser (Linear RGD) peptide concentrations varied between 0.25 and $0.5 \mathrm{mM}$ to control cell adhesion. Vascular endothelial growth factor (VEGF-A-165; R\&D Systems, Minneapolis, MN) concentrations included in endothelial maintenance medium varied between 0,5 , and $10 \mathrm{ng} / \mathrm{mL}$.
FIG. 1. Organotypic culture model system for characterizing chemical effects on NVU models. (A) The organotypic culture model comprises iPSC-derived ECs, iPSC-derived ACs and PCs cultured on an instructive PEG hydrogel that guides assembly of capillarylike networks. Models are photographed through epifluorescence microscopy after 24-hour culture in 96-well plates and analyzed for morphological changes that resulted from chemical exposure. Scale bar: $0.5 \mathrm{~mm}$. Red: ECs. Green: ACs. (B) PEG hydrogels are composed of $20 \mathrm{kDa}, 8$-arm, norbornene-modified PEG molecules that are polymerized through thiol-ene reactions with cell adhesion molecules and cell-degradable crosslinking molecules. The resulting polymer network forms a hydrated free-standing gel. ACs, astrocytes; ECs, endothelial cells; iPSC, induced pluripotent stem cell; NVU, neurovascular unit; PCs, pericytes; PEG, poly(ethylene glycol). Color images are available online.
A

Seeding EC, PC, AC onto PEG hydrogel surfaces

\begin{tabular}{|l|l|l|}
\hline Endothelial Cells (EC) & Pericytes (PC) & Astrocytes (AC) \\
\hline $\begin{array}{l}\text { iPSC-derived ECs } \\
\text { (CDI) }\end{array}$ & $\begin{array}{l}\text { Human Brain- } \\
\text { derived Pericytes } \\
\text { (ScienCell) }\end{array}$ & $\begin{array}{l}\text { iPSC-derived } \\
\text { ACs (CDI) }\end{array}$ \\
\hline
\end{tabular}

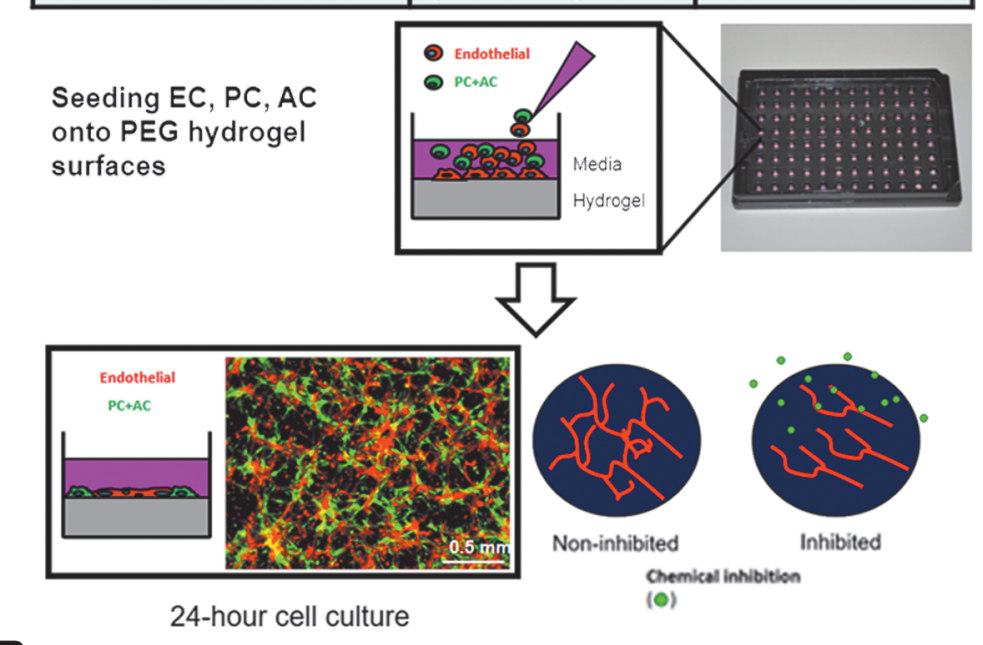

B

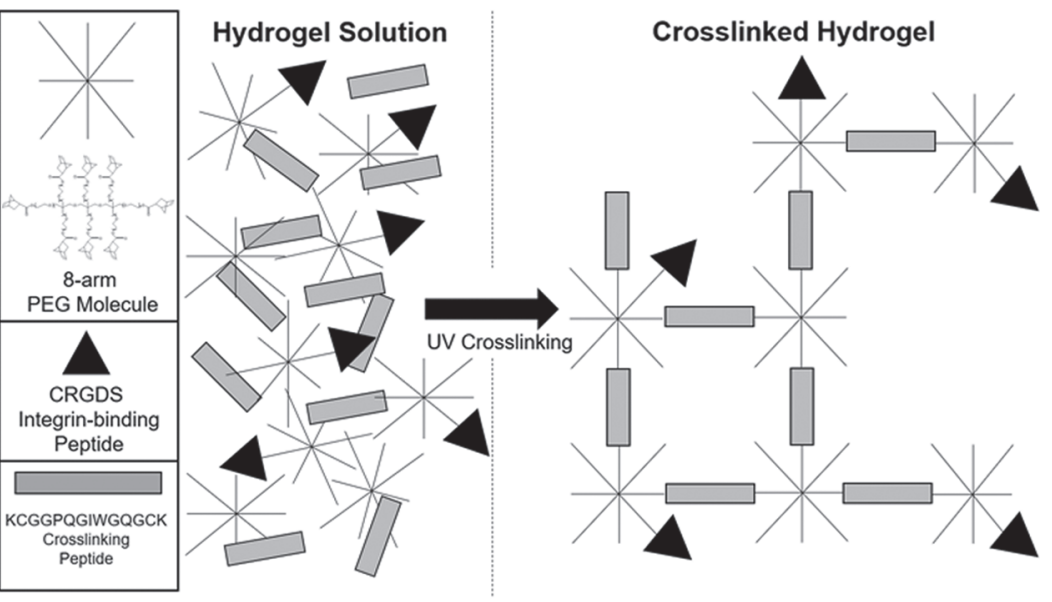



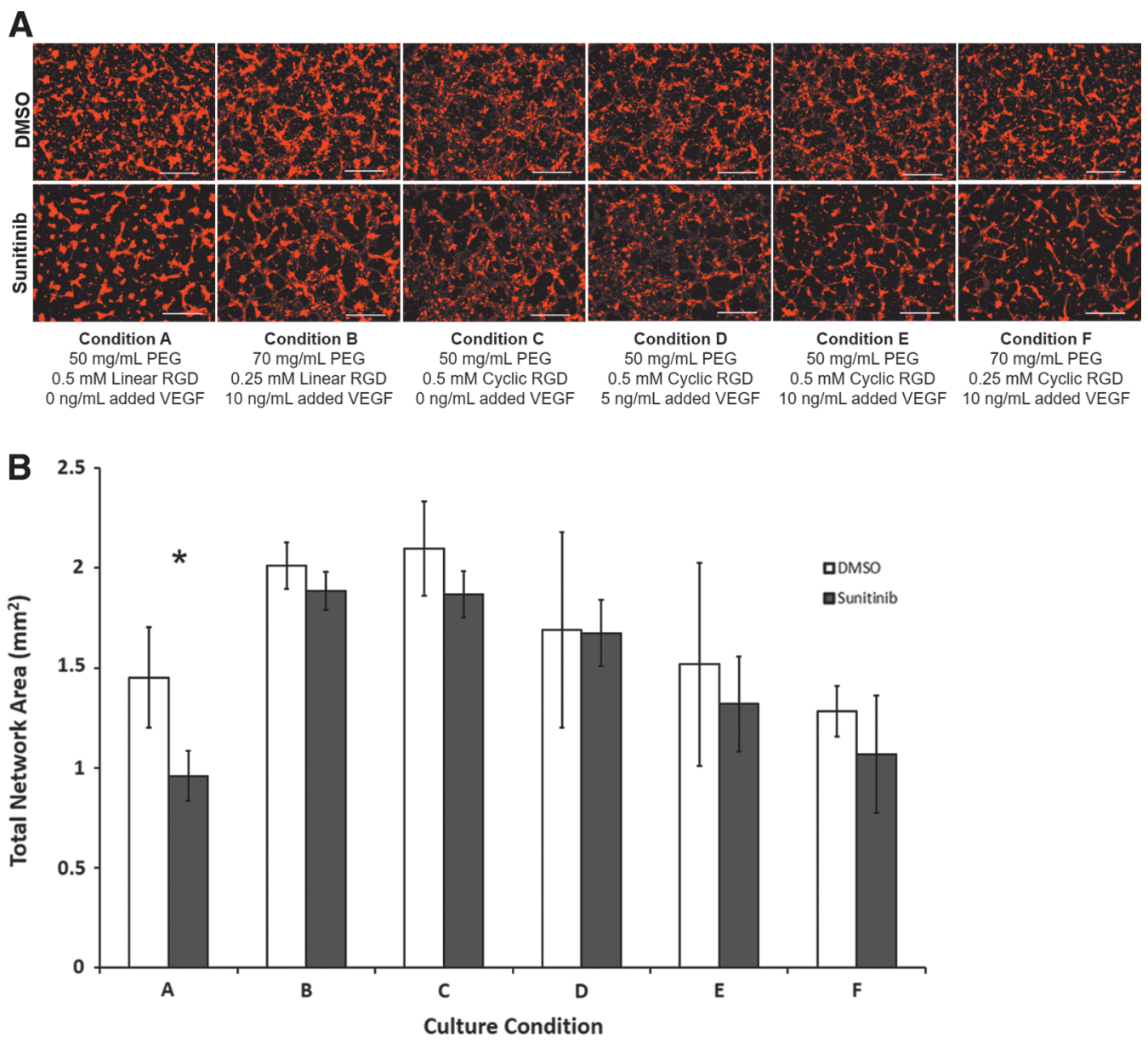

FIG. 2. PEG hydrogels guide self-assembly of ECs into capillary-like networks. (A) Six cell culture conditions were shown to enable endothelial network formation by ECs using $0.5 \mathrm{mM}$ adhesion peptide or less in each hydrogel. Scale bar: $0.5 \mathrm{~mm}$. (B) Condition A showed significant quantifiable effects of sunitinib treatment on total network area, in the form of a low initial network area and the greatest decrease in network area by sunitinib treatment of the tested culture conditions. ${ }^{*} p<0.05$ between DMSO and sunitinib treatments, Sidak's multiple comparisons test. Experimental replicates: A, B: DMSO and sunitinib-8. C, E, F: DMSO and sunitinib-6. D: DMSO_-3. D: sunitinib-4. DMSO, dimethyl sulfoxide. Color images are available online.

Cell Tracker Red-stained ECs were rinsed with $1 \times$ phosphate-buffered saline (PBS). Cells were passaged by incubating in $0.25 \%$ trypsin/EDTA $1 \times$ solution (Thermo Fisher) for 5 minutes, recovering the cells using Vasculife supplemented with $10 \%$ FBS, centrifuging the cells for 5 minutes at 1200 RPM, and resuspending the cell pellets in endothelial maintenance medium supplemented with 0,5 , or $10 \mathrm{ng} / \mathrm{mL}$ additional VEGF as shown in Figure 2A. Supernatant medium was removed from the assembled arrays and replaced with the resuspended cell solution.

Before cell seeding, PEG hydrogel plates were brought to room temperature and $0.1 \mathrm{M}$ PBS was aspirated and replaced with $35 \mu \mathrm{L}$ endothelial maintenance medium. Afterward, cell suspensions in endothelial maintenance medium were added as $35 \mu \mathrm{L}$ volumes to seed 22,500 ECs into each well. After seeding, the assembled constructs were transferred to a $37^{\circ} \mathrm{C}$ incubator and left undisturbed for 24 hours on PEG hydrogels to match the intended 24-hour incubation period of the toxicity assay. After incubation, the arrays were photographed by epifluorescence microscopy using a Nikon TIEclipse microscope. After photography, cells were fixed through 30-minute incubation in $10 \%$ buffered formalin.
The condition chosen for further NVU model development consisted of PEG hydrogels containing $2.5 \mathrm{mM}$ PEGNB, $5 \mathrm{mM}$ MMPDP, and $0.5 \mathrm{mM}$ Linear RGD. In addition, no extra VEGF was added to endothelial maintenance medium.

\section{Determining coculture ratios for NVU model}

After having chosen the desired NVU culture condition, PCs and ACs were added individually, or in combination, with ECs to determine their ability to interact with ECs and affect on capillary network formation within 24 hours of seeding. Cell Tracker Red-stained ECs and either Cell Tracker Green-stained PCs or ACs were rinsed with $1 \times$ PBS. Cells were passaged by incubating $0.25 \%$ trypsin/ EDTA $1 \times$ solution (Thermo Fisher) for 5 minutes, quenching the enzyme through addition of Vasculife supplemented with $10 \%$ FBS, centrifuging the cells for 5 minutes at 1200 RPM, and resuspending the cell pellets in endothelial maintenance medium.

Here, cells were seeded onto hydrogel arrays at densities of 22,500 ECs and either PCs (280-4500 PCs, which corresponds to $1: 80-1: 5$ co-culture ratios of PCs:ECs) or ACs 
(1400-22,500 ACs, which cooresponds to 1:16-1:1 coculture ratios of ACs:ECs) (Fig. 3). After seeding, the assembled constructs were transferred to a $37^{\circ} \mathrm{C}$ incubator and left undisturbed for 24 hours. After incubation, the arrays were photographed by epifluorescence microscopy using a Nikon TI-Eclipse microscope. After photography, cells were fixed through 30-minute incubation in $10 \%$ buffered formalin.

After this, all three cell types were combined together at all possible ratios within the ranges of PCs or ACs added to ECs earlier. In these situations, either PCs or ACs were stained using Cell Tracker Green, but not both cell types simultaneously. Cell seeding onto PEG hydrogel arrays was performed as previously described. In this study, each well contained 22,500 ECs, 280-4500 PCs, and 1400-22,500 ACs depending on desired ratios. Cells were left undisturbed for 24 hours, after which the NVU networks were photographed by epifluorescence microscopy using a Nikon Eclipse microscope. After photography, cells were fixed through 30minute incubation in $10 \%$ buffered formalin. The final NVU coculture ratio chosen for further experimentation was a coculture ratio of $100 \mathrm{ECs}: 5 \mathrm{PCs}: 13 \mathrm{ACs}$, which is a seeding density of 22,500 ECs, 1125 PCs, and 2183 ACs per well.

To test network stability after 72 hours of culture, $70 \mu \mathrm{L}$ endothelial maintenance medium was exchanged from each well 24 hours after seeding and 72 hours after seeding. Images were taken of wells once every 24 hours (Supplementary Fig. S1). Refer to Supplementary Methods for
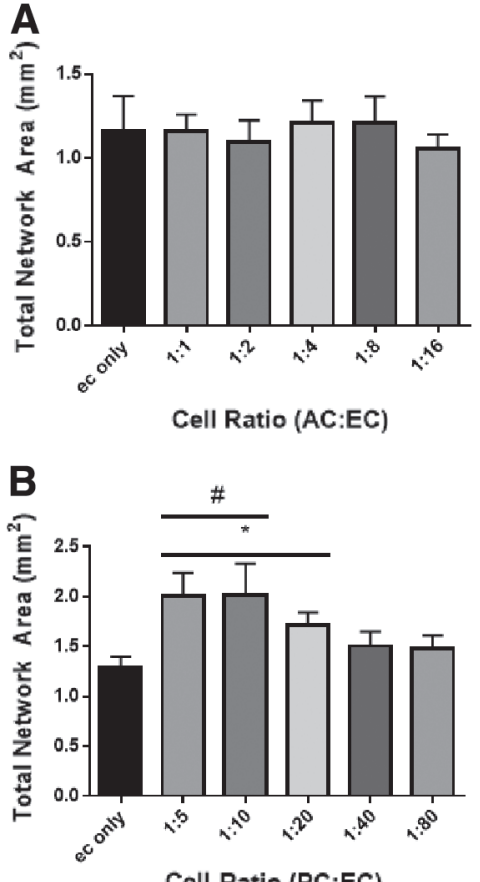

Cell Ratio (PC:EC)

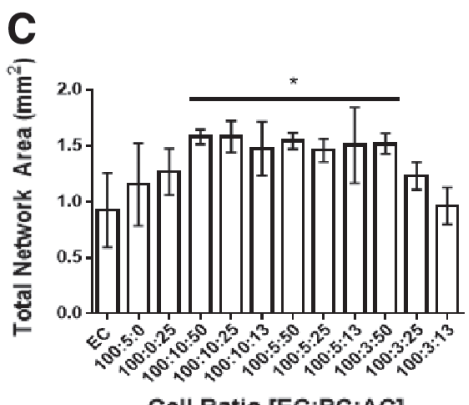

Cell Ratio [EC:PC:AC]

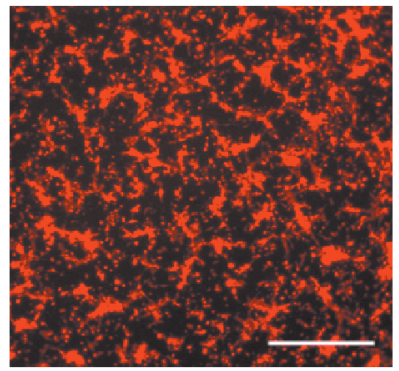

EC only

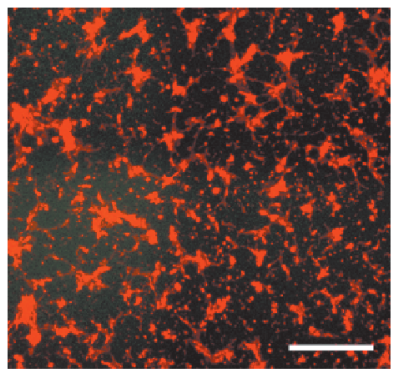

EC only

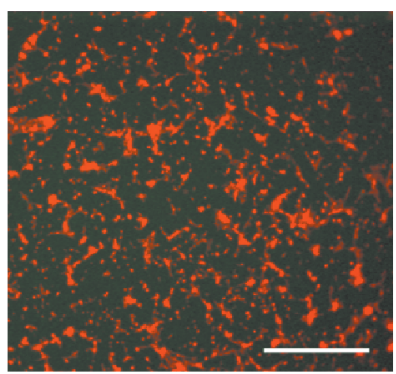

EC Only

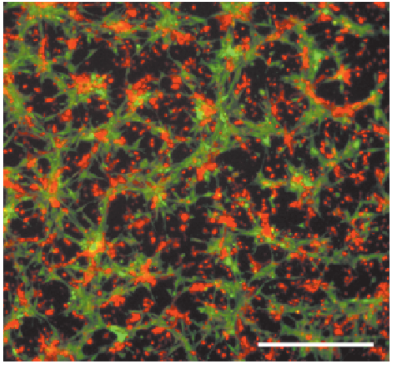

$1 \mathrm{AC}: 2 \mathrm{EC}$

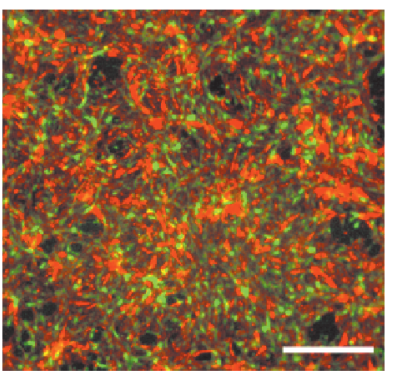

$1 \mathrm{PC}: 5 \mathrm{EC}$

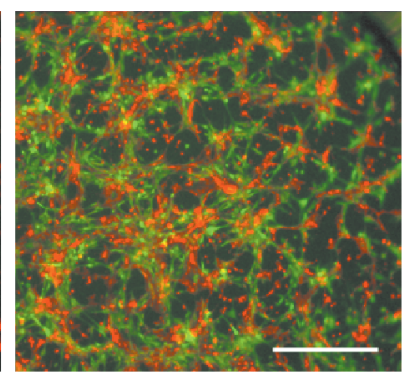

$100 \mathrm{EC}, 10 \mathrm{PC}, 50 \mathrm{AC}$

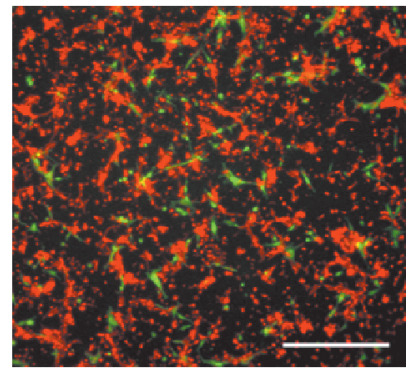

$1 \mathrm{AC}: 16 \mathrm{EC}$

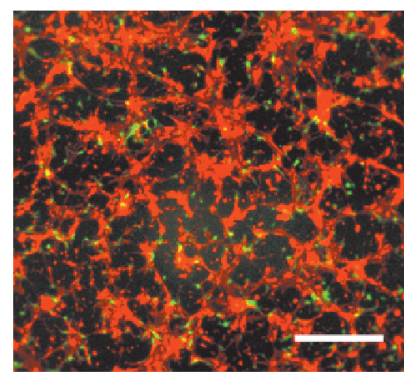

$1 \mathrm{PC}: 20 \mathrm{EC}$

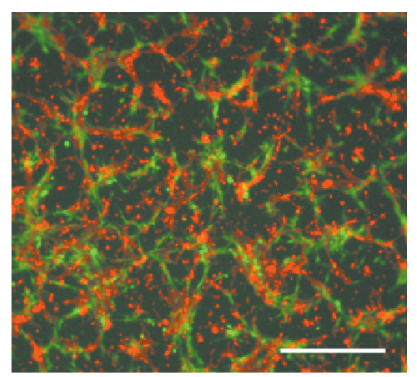

$100 \mathrm{EC}, 5 \mathrm{PC}, 25 \mathrm{AC}$

FIG. 3. Coculture of ECs with PCs and ACs. (A) Dual coculture of ECs with ACs. AC density in coculture varied between 1:1 and 1:16 ratios. The presence of ACs alone did not significantly affect endothelial network area. Scale bar: $0.5 \mathrm{~mm}$. Red: ECs. Green: ACs. (B) Dual coculture of ECs with PCs. PC density in the coculture varied between 1:80 and 1:5 PCs:ECs ratios. A PCs:ECs ratio of 1:20 saw a significant increase in endothelial network area compared with EC-only controls, indicating increased coverage of the hydrogel with more robust vascular networks. Higher PC densities-1:5 and 1:10 PCs:ECs ratios - saw the formation of confluent cell sheets. Scale bar: $0.5 \mathrm{~mm}$. Red: ECs. Green: PCs. (C) Triple coculture of ECs, PCs, and ACs. A number of conditions resulted in a significant increase in network area compared with an EC-only control, but without the formation of confluent cell sheets. Scale bar: $0.5 \mathrm{~mm}$. Red: ECs. Green: ACs. ${ }^{*} p<0.05$ compared with EConly controls, Dunnett's multiple comparisons test. "Conditions where dual coculture formed confluent cell sheets. Color images are available online. 
information on confirming the response of cocultures to receptor tyrosine kinase inhibition and oxidative stress.

\section{Image analysis}

Fluorescent cell images were analyzed using Nikon NIS Elements AR (version 5.02.00, 64-bit; Nikon). Endothelial network formation was quantified and compared using the total endothelial network area identified by NIS Elements software, with minimum size cutoffs (Supplementary Fig. S2A). Networks with disrupted cell-cell connections were identified as having low network area, whereas cocultures forming confluent cell sheets rather than elongated network structures were identified as having significantly higher areas than controls. Ideal networks were identified as having a moderate area compared with the aforementioned scenarios.

Colocalization between ECs and PCs or ACs (ECs/PCs colocalization, ECs/ACs colocalization, respectively) was performed by generating Mander's Overlap Coefficients to represent the overlap between separate color channels per cell type (Supplementary Fig. S2B). Increased colocalization indicated greater degrees of cell-cell interaction and communication between the ECs, PCs, and ACs in the cocultures.

Image skeletonization was performed using Nikon NIS Elements AR skeletonization algorithms (version 5.02.00, 64-bit; Nikon), after converting images to binary, closing holes in structures, and eroding them. Skeletonized images were pruned to remove extraneous branches that represented image artifacts (Supplementary Fig. S3). On Matrigel conditions, an additional function to connect lose ends was applied to portray skeletons of overarching Matrigel networks rather than detailing defects within cell sheets and structures. The number of intersections in each skeletonized image was quantified as a measure of network meshing density in MATLAB (R2017a version 9.2.0.538062, 64-bit; Mathworks). Code and instructions are included in Supplementary Data File S1 (Supplementary Fig. S2C).

Protrusion measurements were performed using MATLAB (R2017a version 9.2.0.538062, 64-bit; Mathworks). Code and instructions are included in Supplementary Data File S2 using methods similar to Iwata et al., ${ }^{46}$ where image filtration methods isolate protrusions and find network features. Both skeletonization and protrusion images were indications of the cells' ability to migrate and generate networks of elongated structures rather than rounded cell clusters of confluent cell sheets. These features were then quantified for area (Supplementary Fig. S2D). Skeletonized images and images for protrusion analysis were transformed into stacked TIF images in ImageJ (version 1.46r; National Institutes of Health $[\mathrm{NIH}]$ ) before analysis in MATLAB. Each color channel, except in colocalization, was analyzed as separate channels.

\section{Screening predicted vascular inhibitors from the ToxCast library}

To identify potential vascular inhibitors from a library of chemical compounds, 38 predicted vascular inhibitory chemicals from the ToxCast chemical library were applied to the NVU models and ECs. As in previous screening experiments, these chemicals were provided by the Environmental Protection Agency (EPA) to encompass a range of predicted activity levels, from "inactive" to "strongly active" inhibi- tory compounds based on a predicted vascular disrupting chemical (pVDC) score from the ToxCast in vitro bioactivity profile. ${ }^{32,33,47}$ Chemical activities in the Toxcast database were derived from $>500$ high-throughput assays, which include EC viability, tubulogenesis, as well as angiogenic protein expression and activity. ${ }^{33,48,49}$ Scores $<0.1$ were predicted as inactive and scores $>0.1$ were predicted as active vascular inhibitory chemicals.

The compounds were presented as 53 samples, including replicates. The dimethyl sulfoxide (DMSO) stock solutions from the library were diluted using endothelial maintenance medium at a 1:1000 dilution before their additions into the screening system. Tested chemicals were added to the system during cell seeding.

Endothelial network inhibition was evaluated similarly to previous screens ${ }^{33}$ by quantifying the total area of endothelial networks treated with unknown compounds and comparing them to mean total areas of noninhibited control networks treated with vehicle only ( $0.2 \%$ DMSO). Networks with areas $>2$ standard deviations (SDs) away from the mean were identified as being inhibited by a candidate compound. ${ }^{33}$ In addition, ECs/PCs colocalization and ECs/ACs colocalization were quantified to screen for chemically mediated changes to ECs/PCs and ECs/ACs interactions, specifically the degree to which PCs and ACs integrated into the vascular network. Networks with coefficients $>2$ SDs away from the DMSO control mean were identified as being inhibited by a candidate compound. The criterion of 2 SDs was adequate for balancing specificity and sensitivity of previous iterations of the vascular screen. ${ }^{33}$

All tests were performed in triplicate and compounds that resulted in inhibition for a majority of replicates were counted as inhibitors. These were given a binary output of either 1 for inhibited conditions or 0 for uninhibited conditions. Compounds identified as inhibitors in a majority of the triplicate plates were counted as inhibitors in the PEG hydrogel and Matrigel systems. To select chemicals for future characterization, all chemicals identified as vascular inhibitors were ranked according to the consistency of detection across six trials for ECs area, and three trials for ECs/PCs and ECs/ACs colocalization. For ECs area, chemicals were categorized as "low," "medium," "high," and "critical" priority based on whether they were detected as inhibitors in three out of six, four out of six, five out of six, and six out of six trials, respectively. For ECs/PCs and ECs/ACs colocalization, chemicals were categorized as "high" and "critical" priority based on whether they were detected as inhibitors in two out of three or three out of three trials, respectively. All chemicals ranked as "critical" priority in any category were chosen as subjects for use in dose-response testing.

\section{Dose-response curve generation}

PEG hydrogel and Matrigel-based screening arrays were generated for dose-response curve generation. In each 96well plate, each chemical was applied to the NVU models in eight dosing concentrations, serially diluted 1:2 between each condition, $n=3$ wells for each concentration. Tested chemicals were added to the system during cell seeding. Dosing ranges were chosen after inspection of known active concentration $50\left(\mathrm{AC}_{50}\right)$ values archived in the EPA Toxcast Forcaster Database. $^{50}$ 
Fluazinam and reserpine were dosed in a concentration range of $0.16-20 \mu \mathrm{M}$. 5HPP33 was dosed in a concentration range of $0.31-40 \mu \mathrm{M}$. 4-Nonylphenol branched, disulfiram, phenolphthalein, pyridaben, quercetin, and triclocarban were dosed in a concentration range of $0.63-80 \mu \mathrm{M}$. 1Hydroxypyrene was dosed in a concentration range of 1.3$160 \mu \mathrm{M}$. Cell densities were identical in PEG hydrogel and Matrigel-based screening arrays. All curves were generated for at least two technical replicates. Any conditions wherein image artifacts interfered with analysis, including chemical autofluorescence at high doses, abnormally low cell seeding densities, and biphasic effects at maximum doses, were excluded from the dose-response curve generation. Refer to Supplementary Methods for information on testing dosedependent cytotoxicity of select chemicals.

\section{Statistics}

All statistical analyses were performed with Graphpad Prism 6 (version 6.05; Graphpad Software). All data analyzed were unpaired (samples independent of each other). Before conducting multiple comparisons tests, the BrownForsythe test was performed to determine homogeneity of variance between data sets when possible. To compare all data sets to one another, Tukey's multiple comparisons test was used as a single-step multiple comparison procedure to find means significantly different from each other. In addition, Sidak's multiple comparisons test was used to compare equivalent cell seeding conditions treated with differing chemicals. To compare data sets with a DMSO vehicle control, Dunnett's test was used to find means significantly different from the control. All statistical tests were two-tailed (two-sided test). $p$-Values were as follows: $p$-value $>0.05$ (nonsignificant), $* p<0.05$. Variances between each group of data are represented by the SD.

Sample sizes to ensure adequate power were as follows: initial EC network formation on PEG hydrogels, $n=3-8$ sample replicates; coculture network formation on PEG hydrogels, $n=4-8$ sample replicates; sunitinib malate and hydrogen peroxide tests, $n=3$ sample replicates, for DMSO and PBS controls $n=12$ sample replicates, and 2 technical replicates per experiment; 38 -chemical ToxCast screen, 3 repeated experiments using the EC-only model, 6 repeated experiments using the NVU model; and dose-response curves for epifluorescence microscopy and cytotoxicity assays, $n=3$ sample replicates, and 2 technical replicates per experiment. Samples were excluded from analysis if they were damaged during the testing procedure, or were determined to be outliers through the Grubbs outlier test.

\section{Data availability}

The authors declare that all data supporting the findings of this study are available within the article and its Supplementary Data files or are available from the corresponding author on request.

\section{Results}

\section{EC network formation on PEG hydrogels}

Twelve EC culture environments, characterized by varying VEGF concentrations added to maintenance medium and varying PEG hydrogel properties, were observed in
Nguyen et al. ${ }^{33}$ to enable self-assembly by iPSC-derived ECs into capillary-like networks. Six of those environments, each requiring $<1 \mathrm{mM}$ adhesion peptide included in the PEG hydrogels, were tested here after assembly in $\mu$-plate angiogenesis 96-well plates. Conditions A and B did not generate any hydrogels that were distorted or nonlevel after overnight swelling.

ECs assembled into networks of capillary-like structures when cultured for 24 hours in all six environments (Fig. 2A). Incubation with $40 \mu \mathrm{M}$ sunitinib malate resulted in significant decreases, compared with DMSO controls, in EC network area only when $0.5 \mathrm{mM}$ CRGDS peptide was incorporated into hydrogels containing $50 \mathrm{mg} / \mathrm{mL}$ PEG, and no exogenous VEGF was added to culture medium (culture condition A, Fig. 2B). The effect was not significant on PEG hydrogels containing cyclic RGD.

We selected culture condition A for screening experiments on the basis that it would provide sensitive readouts of endothelial network disruption by chemical exposure. This condition demonstrated a significant effect of sunitinib treatment on decreasing network area. It also generated a lower network area than other conditions, such as B and C, which would allow room for PCs and ACs to improve network area in coculture. All network measurements were taken at 24 hours after seeding, which matches the time frame of chemical screening assays.

\section{EC network area with cocultured PCs and ACs}

ECs were cocultured with either ACs or PCs to determine density-dependent effects of perivascular cell activity on capillary network formation. Cocultures of ECs and ACs did not result in significant changes to endothelial network area (Fig. 3A). In contrast, cocultures of ECs and PCs resulted in increased total endothelial network area with increasing density of PCs (Fig. 3B). A significant increase in area was observed at a 1:20 PCs:ECs coculture ratio while not promoting the formation of confluent cell sheets. Higher densities of PCs would motivate the generation of confluent cell sheets rather than capillary-like endothelial networks and were considered inappropriate for use in a capillary network formation assay. It was known that PCs were capable of increasing EC survival and tight junction stability, ${ }^{51}$ and we were able to observe this interaction in the form of EC monolayer formation here.

ECs, PCs, and ACs were cocultured simultaneously to determine density-dependent effects that the combination of PCs and ACs would have on capillary network formation. The presence of ACs in the NVU model prevented the formation of confluent cell sheets that would have previously occurred in the presence of high (1:5 and 1:10 PCs:ECs ratios) PC densities. PCs, ACs, and ECs are known to interact with each other to stabilize capillary networks, the ACs primarily modulating vessel activity through PC communication, ${ }^{17,18}$ and we were likely seeing that interaction in the form of vessel stabilization here. Because of this effect, a total of $7 \mathrm{NVU}$ coculture ratios resulted in significantly increased endothelial network area compared with an EConly control, each occurring without the formation of confluent cell sheets (Fig. 3C). We selected the triple coculture with the lowest density of perivascular cells - a 13:5:100 ACs:PCs:ECs ratio-in culture environment $\mathrm{A}$ as the 
NVU model to characterize chemical activity. Refer to Supplementary Results for information on confirming the response of cocultures to receptor tyrosine kinase inhibition and oxidative stress (Supplementary Fig. S4).

\section{Initial screening and prioritization of neurovascular disrupting chemicals}

We treated the NVU model and an equivalent EC monoculture models in identical culture environments with a panel of 38 pVDCs supplied by the EPA. These tests were performed to narrow an initial library of chemicals down to a smaller selection of critical priority chemicals to be used in doseresponse curve generation. These chemicals were previously curated to have a range of antiangiogenic effects on ECs. ${ }^{33}$ Chemicals with $p$ VDC scores of $\geq 0.1$ were expected to have high antiangiogenic activity, and chemicals with pVDC scores $<0.1$ were expected to have low antiangiogenic activity.

Of the chemicals screened, the EC-only culture detected 9 out of the 38 chemicals as significant effectors of the network area. The NVU model detected 24 out of 38 chemicals as significant effectors of the network area (Fig. 4A, B). In the case of the NVU model, the number of detected chemicals spanned the entire range of predicted antiangiogenic activity (pVDC score), rather than only chemicals with high pVDC scores. The screens were conducted using six technical replicates total for the NVU model, and three technical replicates for the EC-monoculture model. ECs were labeled using Cell Tracker Red in each trial. In the NVU models, ACs were labeled using Cell Tracker Green in three out of six trials, and PCs were labeled using Cell Tracker Green in the remaining three out of six trials.

To determine which individual cell types in the NVU model were affected by the pVDC panel, a colocalization measurement was conducted to quantify the overlap between either ACs or PCs with EC networks (ECs/ACs colocalization and ECs/PCs colocalization, respectively) (Supplementary Fig. S2B). ECs/PCs colocalization resulted in 11 out of 38 chemicals being detected as effective chemicals (Fig. 4C), whereas ECs/ACs colocalization resulted in 8 out of 38 chemicals being detected as effective chemicals (Fig. 4D). In both measurements, all detected chemicals had a pVDC score of $\geq 0.26$, in contrast to the EC area result of chemicals with pVDC scores as low as 0 being detected as potential disrupting chemicals.

To select specific chemicals for further characterization, the chemicals were sorted into categories of low, medium, high, and critical priority based on consistency of detected changes to endothelial network area, ECs/ACs colocalization, and ECs/PCs colocalization across the six screening trials. Chemicals that significantly changed endothelial network area in three, four, five, or six out of six trials were categorized as "low," "medium," high," and "critical" priority chemicals, respectively, in the context of targeting ECs behavior. In the case of the ECs/ACs and ECs/PCs colocalization, chemicals that were detected as hits in two or three out of three trials were categorized as "high" or "critical" priority chemicals, respectively, in the context of targeting $\mathrm{AC}$ and $\mathrm{PC}$ behaviors.

Ten chemicals emerged as "critical" priority chemicals from combined endothelial network area, ECs/ACs colocalization, and ECs/PCs colocalization results. These chemi- cals: 1-hydroxypyrene, 4-nonylphenol branched, 5HPP33, disulfiram, fluazinam, phenolphthalein, pyridaben, quercetin, triclocarban, and reserpine, were selected for dose-response characterization (Fig. 4E).

\section{Dose-response curves and signature effects of critical priority chemicals}

To characterize the effects of chemical exposure on the NVU model, nine morphological measurements were taken based on features pertaining to the individual cell types included in the model. Dose-response curves were generated for each of the morphological measurements (Supplementary Fig. S2). The resulting sets of $\mathrm{AC}_{50}$ values derived from the curves were unique to each chemical applied to the model (Table 1, all representative dose-response curves in Supplementary Fig. S5).

Decreases in the majority of the nine morphological metrics are an indication that the NVU cells were adopting rounded nonadhesive morphology as a result of chemical exposure. Fluazinam, 5HPP33, and triclocarban significantly decreased the magnitude of most of the metrics analyzed in the study. In the case of phenolphthalein, we observed similar nonadhesive phenotypes as fluazinam and 5HPP33 in ECs. However, phenolphthalein did not generate usable $\mathrm{AC}_{50}$ values in a majority of metrics pertinent to $\mathrm{ACs}$ and PCs. This may suggest that ACs and PCs were less sensitive than ECs. Disulfiram generated usable $\mathrm{AC}_{50}$ values concerning $\mathrm{EC}$ protrusions, but $\mathrm{AC}$ morphology required higher disulfiram doses for the generation of $\mathrm{AC}_{50}$ values. Pyridaben uniquely increased network area and the number of nodes in skeletonized images of the EC networks, indicating a reorganization of cells into tightly meshed networks rather than disruption.

1-Hydroxypyrene required very high doses exceeding, in many cases, $50 \mu \mathrm{M}$ to generate usable $\mathrm{AC}_{50}$ values. 4Nonylphenol branched and reserpine largely did not generate usable $\mathrm{AC}_{50}$ values, even though $20 \mu \mathrm{M}$ doses were sufficient for causing a significant change in screening parameters for initial detection (Fig. 4). These effects included changing $\mathrm{EC} / \mathrm{PC}$ colocalization through 4-nonylphenol branched, as well as EC/AC and EC/PC colocalization through reserpine. In the cases of reserpine and quercetin, chemical autofluorescence may have interfered with morphological measurements related to ACs and PCs (Supplementary Fig. S6). However, quercetin did impact endothelial network formation, particularly that it impacted network area without changing network protrusions as its antiangiogenic effect. Refer to Supplementary Results for confirmation of noncytotoxic chemical mechanisms observed in this study (Supplementary Fig. S7).

The $\mathrm{AC}_{50}$ values found in our dose-response experiments were compared with the median and range of human vascular and human brain $\mathrm{AC}_{50}$ values found in the EPA ToxCast Database $^{50}$ (Fig. 5 and Supplementary Fig. S5). The chemicals that generated $\mathrm{AC}_{50}$ values closest to the ToxCast median include quercetin, fluazinam, and pyridaben. Chemicals that were less accurate but still generated $\mathrm{AC}_{50}$ values within the range of the ToxCast assays included disulfiram, phenolphthalein, triclocarban, and 5HPP-33. Chemicals that generated $\mathrm{AC}_{50}$ values outside of the range of the ToxCast assays include reserpine, 1-hydroxypyrene, and 4nonylphenol branched. 
FIG. 4. Ranking and prioritization of vascular disrupting chemicals detected from initial screening experiments using the NVU model. (A) Comparison of screening results using an ECs monoculture and the NVU model. Of the EPA predicted vascular disrupting chemical panel, the NVU model detected a greater number of chemicals than the ECs monoculture. Results were based on changes to endothelial network area compared with DMSO controls. (B) Ranking of chemicals detected in the NVU model based on consistency of detection across six trials of endothelial network area, as well as three trials of (C) ECs/PCs colocalization and (D) ECs/ACs colocalization screening experiments. (E) Final list of critical priority chemicals selected for further characterization. Chemicals sorted according to whether they were critical priority chemicals using endothelial network area, ECs/PCs colocalization, or ECs/ACs colocalization as readouts. EPA, Environmental Protection Agency. Color images are available online. Continued

\begin{tabular}{|c|c|c|c|}
\hline Chemical Name: & ECs Alone & NVU Co-culture & pVDC score \\
\hline 1,2,4-Trichlorobenzene & 1 & 1 & 0 \\
\hline Decane & & 1 & 0 \\
\hline Tris(2-chloroethyl) phosphate & & & 0 \\
\hline 1,2,3-Trichloropropane & & 1 & 0.002 \\
\hline Diethanolamine & & 1 & 0.002 \\
\hline Methimazole & & 1 & 0.002 \\
\hline Pymetrozine & & 1 & 0.002 \\
\hline D-Mannitol & & 1 & 0.007 \\
\hline Imazamox & 1 & 1 & 0.007 \\
\hline Methylparaben & & & 0.01 \\
\hline Valproic acid & & 1 & 0.016 \\
\hline 2,4-Diaminotoluene & & & 0.069 \\
\hline Bisphenol A & & & 0.146 \\
\hline Haloperidol & & 1 & 0.177 \\
\hline Tris(2-ethylhexyl) phosphate & & 1 & 0.182 \\
\hline Tris(1,3-dichloro-2-propyl)phosphate & & & 0.188 \\
\hline Cladribine & & & 0.196 \\
\hline TNP-470 & & 1 & 0.238 \\
\hline Oxytetracycline dehydrate & & & 0.26 \\
\hline Celecoxib & & 1 & 0.269 \\
\hline Docusate sodium & & 1 & 0.304 \\
\hline C.I. Solvent Yellow 14 & 1 & 1 & 0.306 \\
\hline Reserpine & & & 0.307 \\
\hline Quercetin & 1 & & 0.309 \\
\hline 5HPP-33 & & 1 & 0.327 \\
\hline Phenolphthalein & 1 & 1 & 0.327 \\
\hline tert-Butylhydroquinone & 1 & 1 & 0.336 \\
\hline Triclocarban & & & 0.362 \\
\hline Triclosan & 1 & & 0.372 \\
\hline Pyridaben & 1 & 1 & 0.379 \\
\hline 1-Hydroxypyrene & & & 0.386 \\
\hline Sodium dodecylbenzenesulfonate & & & 0.429 \\
\hline Disulfiram & 1 & 1 & 0.432 \\
\hline Fluazinam & & 1 & 0.434 \\
\hline Octyl gallate & & 1 & 0.45 \\
\hline Bisphenol AF & & & 0.457 \\
\hline PFOS & & 1 & 0.46 \\
\hline 4-Nonylphenol, branched & & 1 & 0.461 \\
\hline
\end{tabular}

\begin{tabular}{|c|c|c|c|c|c|}
\hline Chemical Name: & Low Priority & Mid Priority & High Priority & Critical Priority & pVDC score \\
\hline 1,2,4-Trichlorobenzene & & 1 & & & 0 \\
\hline Decane & & 1 & & & 0 \\
\hline Tris(2-chloroethyl) phosphate & & & & & 0 \\
\hline 1,2,3-Trichloropropane & & 1 & & & 0.002 \\
\hline Diethanolamine & 1 & & & & 0.002 \\
\hline Methimazole & & 1 & & & 0.002 \\
\hline Pymetrozine & & & 1 & & 0.002 \\
\hline D-Mannitol & & & 1 & & 0.007 \\
\hline Imazamox & & & 1 & & 0.007 \\
\hline Methylparaben & & & & & 0.01 \\
\hline Valproic acid & & 1 & & & 0.016 \\
\hline 2,4-Diaminotoluene & & & & & 0.069 \\
\hline Bisphenol A & & & & & 0.146 \\
\hline Haloperidol & & & 1 & & 0.177 \\
\hline Tris(2-ethylhexyl) phosphate & 1 & & & & 0.182 \\
\hline Tris(1,3-dichloro-2-propyl)phosphate & & & & & 0.188 \\
\hline Cladribine & & & & & 0.196 \\
\hline TNP-470 & & & 1 & & 0.238 \\
\hline Oxytetracycline dihydrate & & & & & 0.26 \\
\hline Celecoxib & & 1 & & & 0.269 \\
\hline Docusate sodium & & 1 & & & 0.304 \\
\hline C.I. Solvent Yellow 14 & 1 & & & & 0.306 \\
\hline Reserpine & & & & & 0.307 \\
\hline Quercetin & & & & & 0.309 \\
\hline $5 \mathrm{HPP}-33$ & 1 & & & & 0.327 \\
\hline Phenolphthalein & & & & 1 & 0.327 \\
\hline tert-Butylhydroquinone & & 1 & & & 0.336 \\
\hline Triclocarban & & & & & 0.362 \\
\hline Triclosan & & & & & 0.372 \\
\hline Pyridaben & & & & 1 & 0.379 \\
\hline 1-Hydroxypyrene & & & & & 0.386 \\
\hline Sodium dodecylbenzenesulfonate & & & & & 0.429 \\
\hline Disulfiram & & & 1 & & 0.432 \\
\hline Fluazinam & 1 & & & & 0.434 \\
\hline Octyl gallate & 1 & & & & 0.45 \\
\hline Bisphenol AF & & & & & 0.457 \\
\hline PFOS & 1 & & & & 0.46 \\
\hline 4-Nonylphenol, branched & 1 & & & & 0.461 \\
\hline
\end{tabular}




\section{C}

\begin{tabular}{|l|l|l|c|}
\hline Chemical Name: & High Priority & Critical Priority & pVDC score \\
\hline 1,2,4-Trichlorobenzene & & & 0 \\
\hline Decane & & & 0 \\
\hline Tris(2-chloroethyl) phosphate & & & 0 \\
\hline $1,2,3-$ Trichloropropane & & & 0.002 \\
\hline Diethanolamine & & & 0.002 \\
\hline Methimazole & & & 0.002 \\
\hline Pymetrozine & & & 0.002 \\
\hline D-Mannitol & & & 0.007 \\
\hline Imazamox & & & 0.007 \\
\hline Methylparaben & & & 0.01 \\
\hline Valproic acid & & & 0.016 \\
\hline 2,4-Diaminotoluene & & & 0.069 \\
\hline Bisphenol A & & & 0.146 \\
\hline Haloperidol & & & 0.177 \\
\hline Tris(2-ethylhexyl) phosphate & & & 0.182 \\
\hline Tris(1,3-dichloro-2-propyl)phosphate & & & 0.188 \\
\hline Cladribine & & & 0.238 \\
\hline TNP-470 & & & 0.26 \\
\hline Oxytetracycline dihydrate & & & 0.269 \\
\hline Celecoxib & & & 0.304 \\
\hline Docusate sodium & & & 0.306 \\
\hline C.I. Solvent Yellow 14 & 1 & & 0.309 \\
\hline Reserpine & & & 0.327 \\
\hline Quercetin & & & 0.327 \\
\hline 5HPP-33 & & & 0.336 \\
\hline Phenolphthalein & & & 0.362 \\
\hline tert-Butylhydroquinone & 1 & & 0.372 \\
\hline Triclocarban & 1 & & 0.379 \\
\hline Triclosan & 1 & & 0.386 \\
\hline Pyridaben & 1 & & 0.429 \\
\hline 1-Hydroxypyrene & & & 0.432 \\
\hline Sodium dodecylbenzenesulfonate & & & 0.45 \\
\hline Disulfiram & 1 & & 0.467 \\
\hline Fluazinam & & & \\
\hline Octyl gallate & & & \\
\hline Bisphenol AF & & & \\
\hline PFOS & & & \\
\hline 4-Nonylphenol, branched & & & \\
\hline
\end{tabular}

\begin{tabular}{|c|c|c|c|c|}
\hline Chemical Name: & $\begin{array}{l}\text { PC NVU } \\
\text { Critical }\end{array}$ & $\begin{array}{l}\text { AC NVU } \\
\text { Critical }\end{array}$ & $\begin{array}{l}\text { EC NVU } \\
\text { Critical }\end{array}$ & $\begin{array}{l}\text { pVDC } \\
\text { score }\end{array}$ \\
\hline 1,2,4-Trichlorobenzene & & & & 0 \\
\hline Decane & & & & 0 \\
\hline Tris(2-chloroethyl) phosphate & & & & 0 \\
\hline 1,2,3-Trichloropropane & & & & 0.002 \\
\hline Diethanolamine & & & & 0.002 \\
\hline Methimazole & & & & 0.002 \\
\hline Pymetrozine & & & & 0.002 \\
\hline D-Mannitol & & & & 0.007 \\
\hline บ Imazamox & & & & 0.007 \\
\hline Methylparaben & & & & 0.01 \\
\hline Valproic acid & & & & 0.016 \\
\hline 2,4-Diaminotoluene & & & & 0.069 \\
\hline Bisphenol A & & & & 0.146 \\
\hline Haloperidol & & & & 0.177 \\
\hline Tris(2-ethylhexyl) phosphate & & & & 0.182 \\
\hline Tris(1,3-dichloro-2-propyl)phosphate & & & & 0.188 \\
\hline Cladribine & & & & 0.196 \\
\hline TNP-470 & & & & 0.238 \\
\hline Oxytetracycline dihydrate & & & & 0.26 \\
\hline Celecoxib & & & & 0.269 \\
\hline \begin{tabular}{|l|} 
Docusate sodium \\
\end{tabular} & & & & 0.304 \\
\hline C.I. Solvent Yellow 14 & & & & 0.306 \\
\hline Reserpine & 1 & 1 & & 0.307 \\
\hline Quercetin & & 1 & & 0.309 \\
\hline 5HPP-33 & & 1 & & 0.327 \\
\hline Phenolphthalein & & & 1 & 0.327 \\
\hline tert-Butylhydroquinone & & & & 0.336 \\
\hline Triclocarban & & 1 & & 0.362 \\
\hline Triclosan & & & & 0.372 \\
\hline Pyridaben & & & 1 & 0.379 \\
\hline 1-Hydroxypyrene & 1 & 1 & & 0.386 \\
\hline Sodium dodecylbenzenesulfonate & & & & 0.429 \\
\hline Disulfiram & & 1 & & 0.432 \\
\hline Fluazinam & 1 & 1 & & 0.434 \\
\hline Octyl gallate & & & & 0.45 \\
\hline Bisphenol AF & & & & 0.457 \\
\hline PFOS & & & & 0.46 \\
\hline 4-Nonylphenol, branched & 1 & & & 0.461 \\
\hline
\end{tabular}

\begin{tabular}{|c|c|c|c|}
\hline D Chemical Name: & High Priority & Critical Priority & pVDC score \\
\hline 1,2,4-Trichlorobenzene & & & 0 \\
\hline \& Decane & & & 0 \\
\hline Tris(2-chloroethyl) phosphate & & & 0 \\
\hline 1,2,3-Trichloropropane & & & 0.002 \\
\hline 5 Diethanolamine & & & 0.002 \\
\hline Methimazole & & & 0.002 \\
\hline Pymetrozine & & & 0.002 \\
\hline D-Mannitol & & & 0.007 \\
\hline Imazamox & & & 0.007 \\
\hline Methylparaben & & & 0.01 \\
\hline \begin{tabular}{|l|l|} 
Valproic acid \\
\end{tabular} & & & 0.016 \\
\hline 2,4-Diaminotoluene & & & 0.069 \\
\hline Bisphenol A & & & 0.146 \\
\hline Haloperidol & & & 0.177 \\
\hline Tris(2-ethylhexyl) phosphate & & & 0.182 \\
\hline Tris(1,3-dichloro-2-propyl)phosphate & & & 0.188 \\
\hline Cladribine & & & 0.196 \\
\hline TNP-470 & & & 0.238 \\
\hline Oxytetracycline dihydrate & 1 & & 0.26 \\
\hline Celecoxib & & & 0.269 \\
\hline \begin{tabular}{|l|l} 
Docusate sodium \\
\end{tabular} & & & 0.304 \\
\hline C.I. Solvent Yellow 14 & & & 0.306 \\
\hline Reserpine & & 1 & 0.307 \\
\hline Quercetin & & 1 & 0.309 \\
\hline $5 \mathrm{HPP}-33$ & & 1 & 0.327 \\
\hline Phenolphthalein & & & 0.327 \\
\hline tert-Butylhydroquinone & & & 0.336 \\
\hline Triclocarban & & 1 & 0.362 \\
\hline Triclosan & & & 0.372 \\
\hline \begin{tabular}{|l|} 
Pyridaben \\
\end{tabular} & & & 0.379 \\
\hline 1-Hydroxypyrene & & 1 & 0.386 \\
\hline Sodium dodecylbenzenesulfonate & & & 0.429 \\
\hline 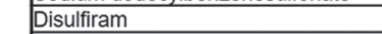 & & 1 & 0.432 \\
\hline Fluazinam & & 1 & 0.434 \\
\hline Octyl gallate & & & 0.45 \\
\hline Bisphenol AF & & & 0.457 \\
\hline PFOS & & & 0.46 \\
\hline 4-Nonylphenol, branched & & & 0.461 \\
\hline
\end{tabular}

\begin{tabular}{|c|c|c|}
\hline ECs Critical & PCs Critical & ACs Critical \\
\hline Phenolphthalein & Reserpine & Reserpine \\
\hline Pyridaben & 1-Hydroxypyrene & 1-Hydroxypyrene \\
\hline & Flazinam & Quercetin \\
\hline & 4-Nonylphenol, Branched & 5HPP-33 \\
\hline & & Triclocarban \\
\hline & & Disulfiram \\
\hline & & Flazinam \\
\hline
\end{tabular}

FIG. 4. (Continued). 


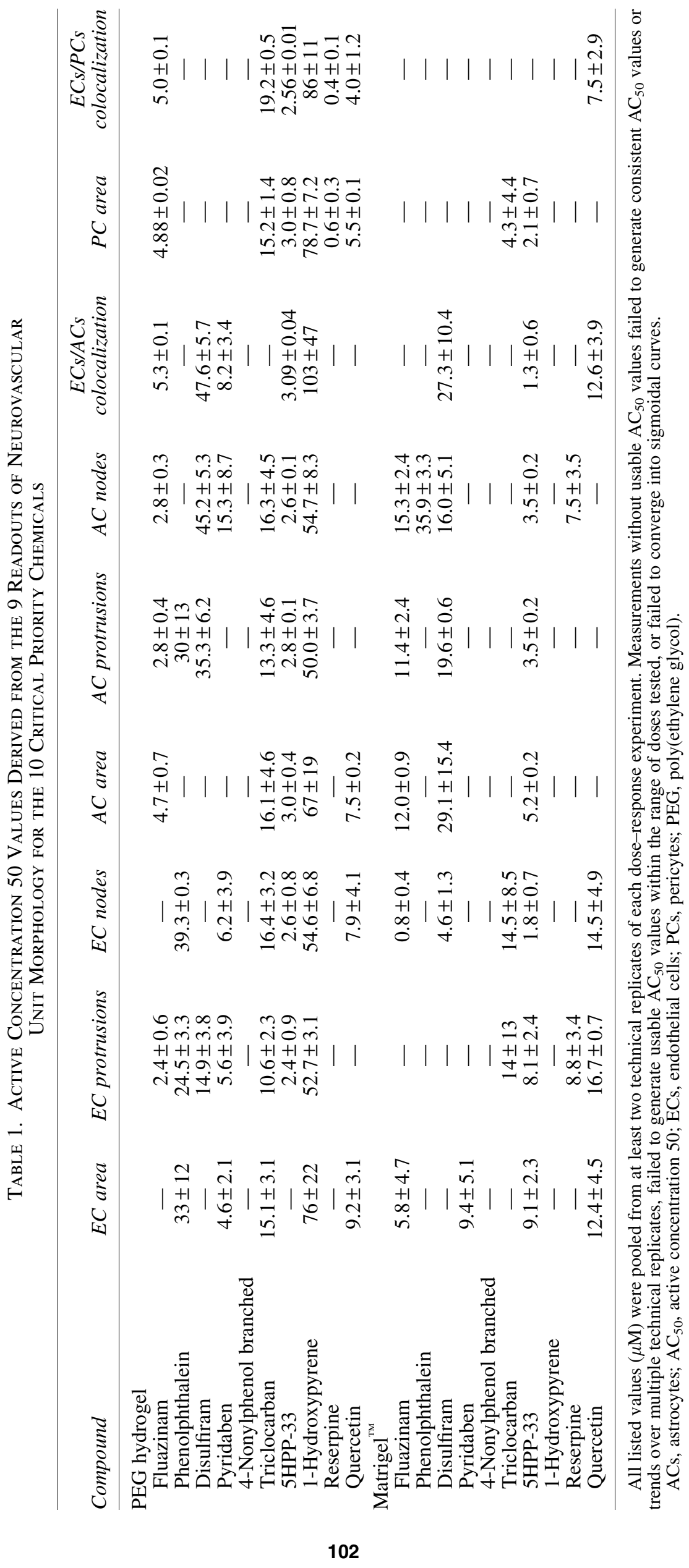



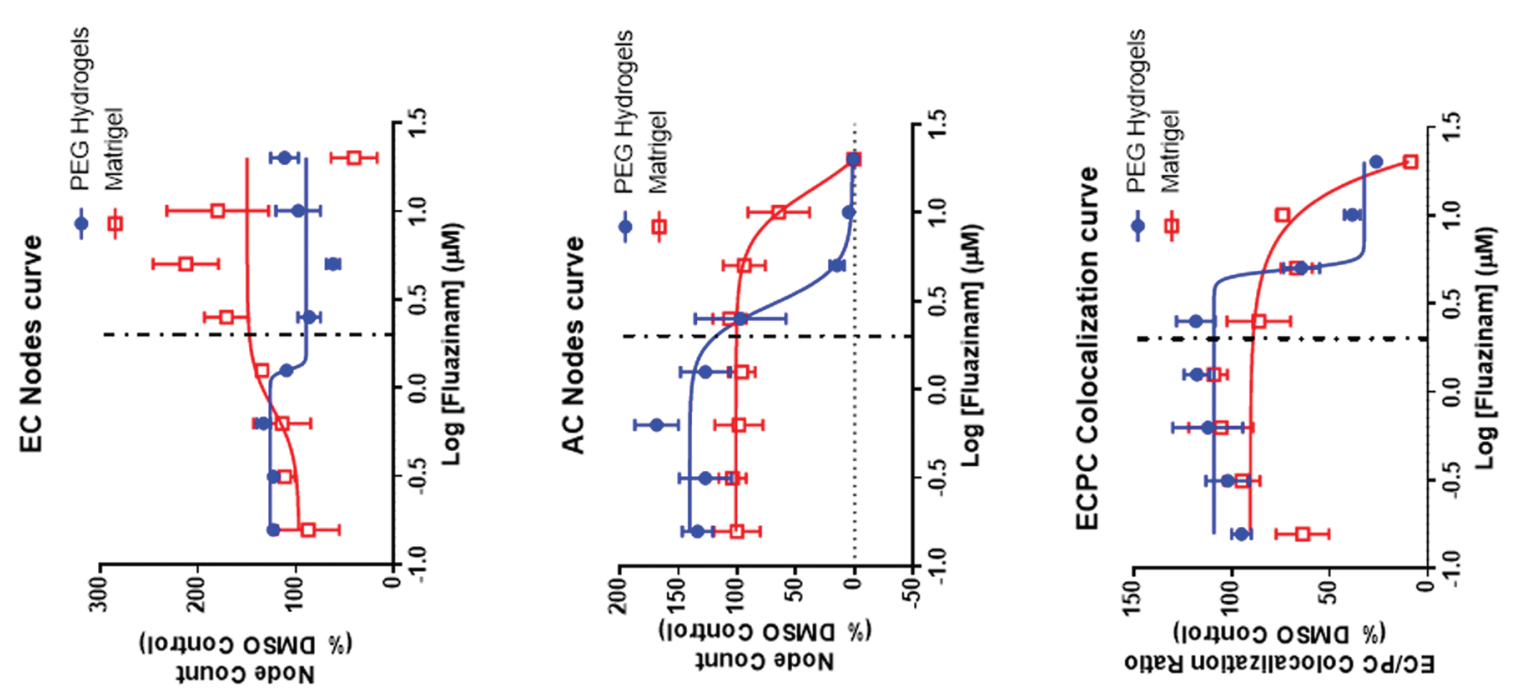

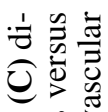
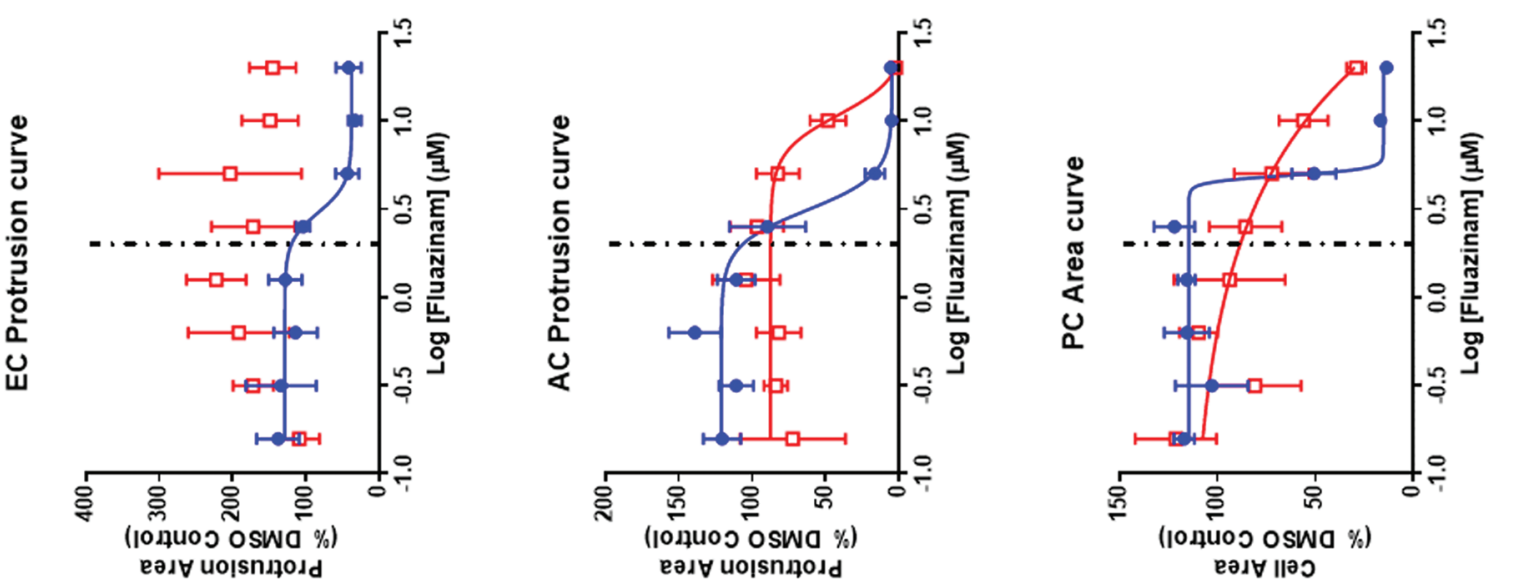

ल․ 空定至 बิํํㅇ 衰瓷

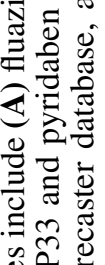

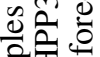
票吉

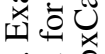
: 氖氙 구ㅇㅝㅡㄹ 늘

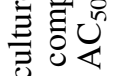
总芯 虽之

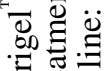
类政
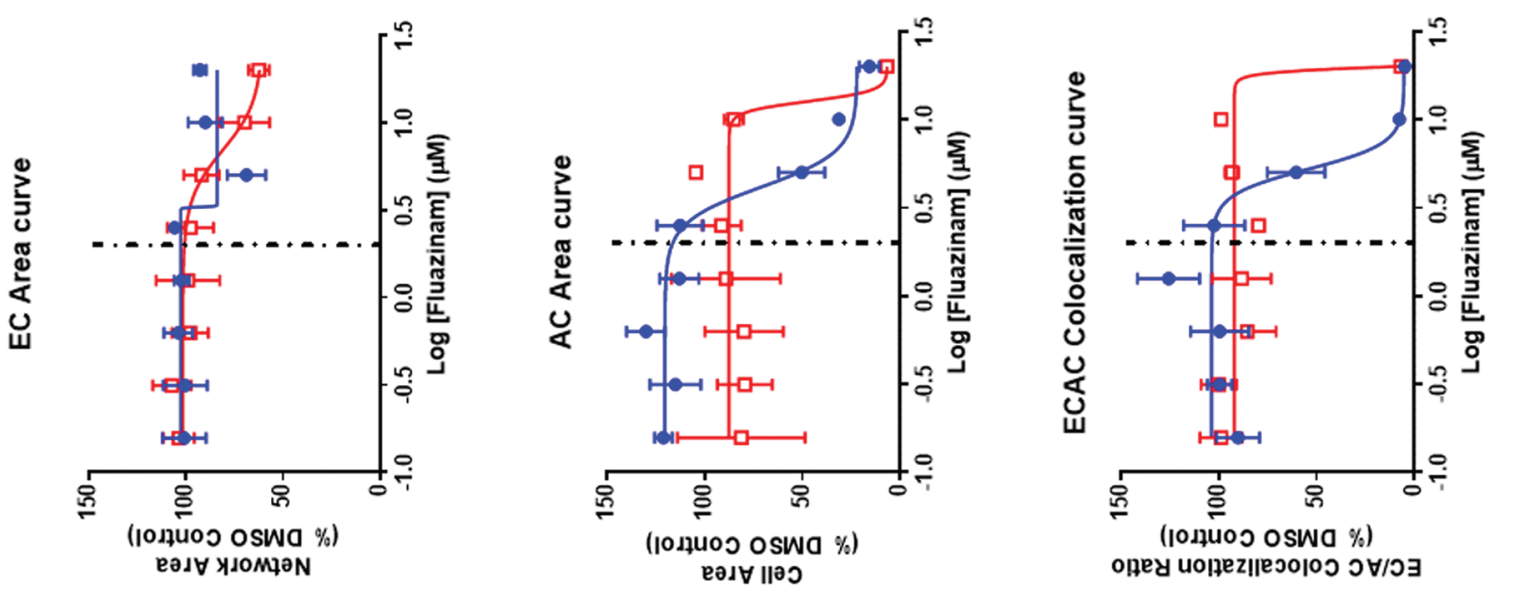

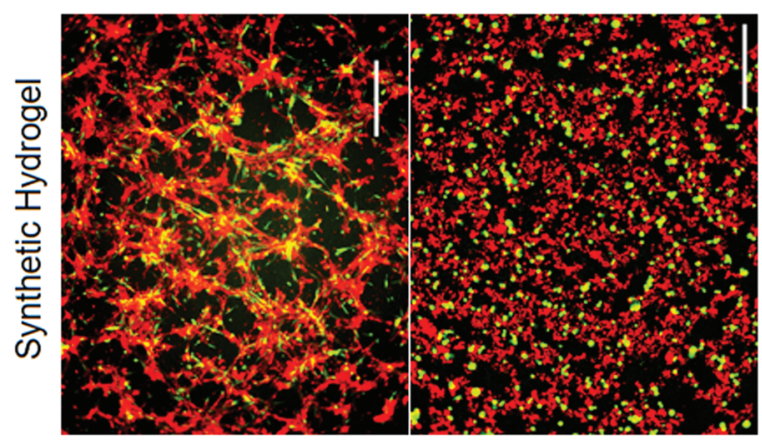

$\varangle \quad$ Oswa \%เ0

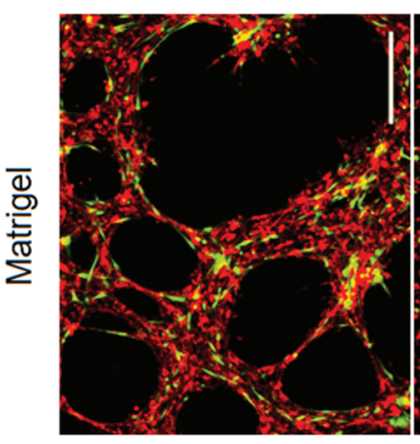

OSWO \%

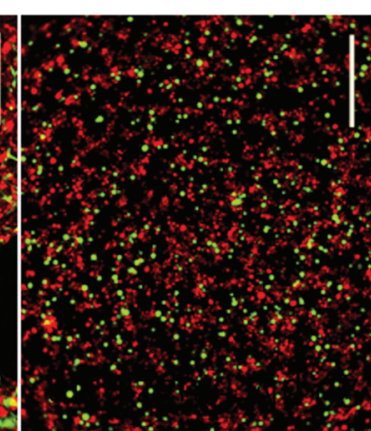

meu!zen! $\exists$ Wrl 02

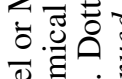
品 tण बै बें

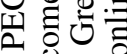

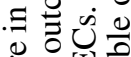
قี่ 징 중 छ 를 : O

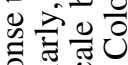
के

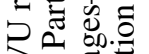
乙焉 4 . 记.

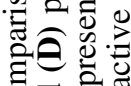

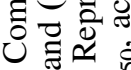

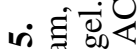
语焉焉 

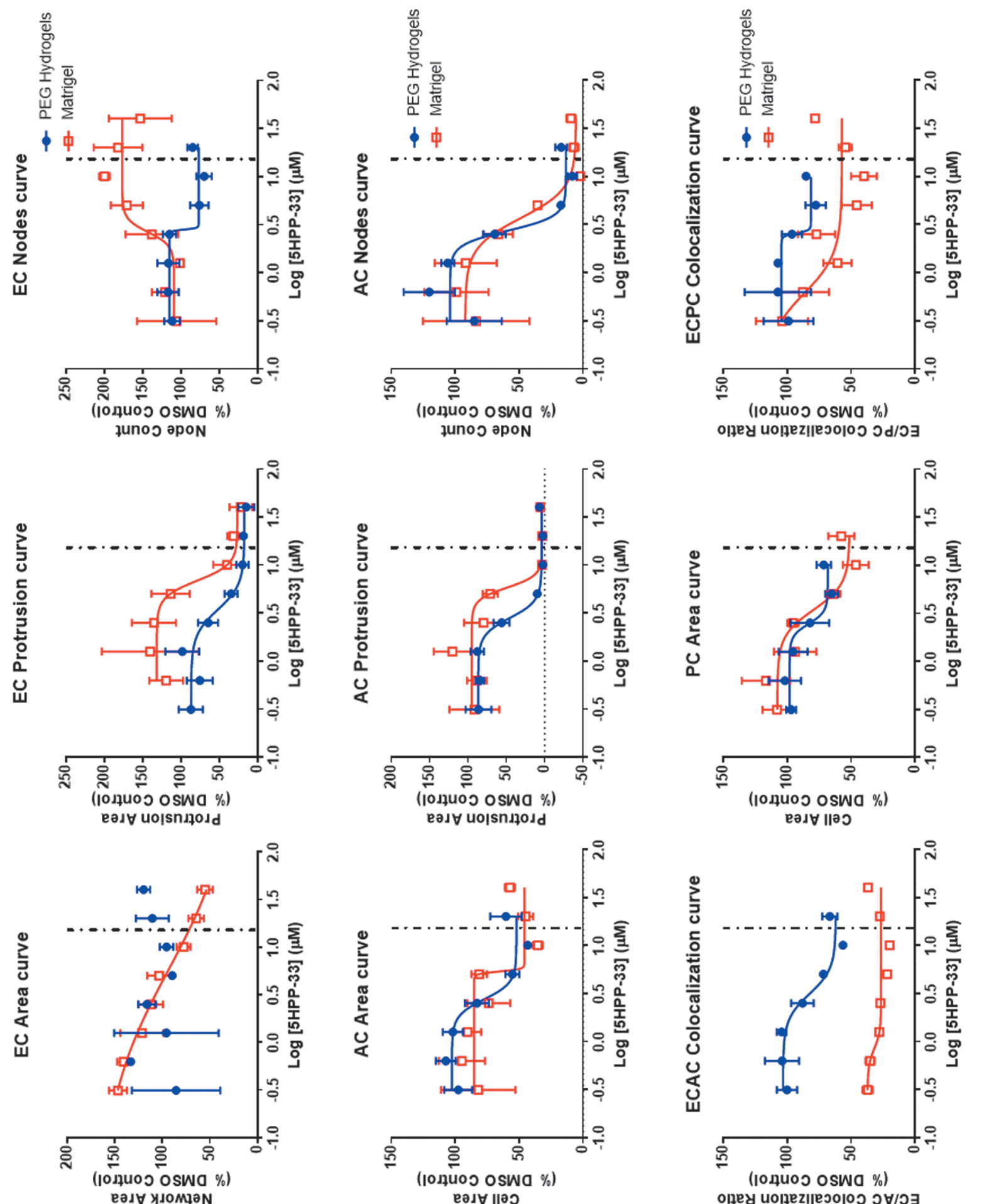

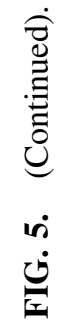
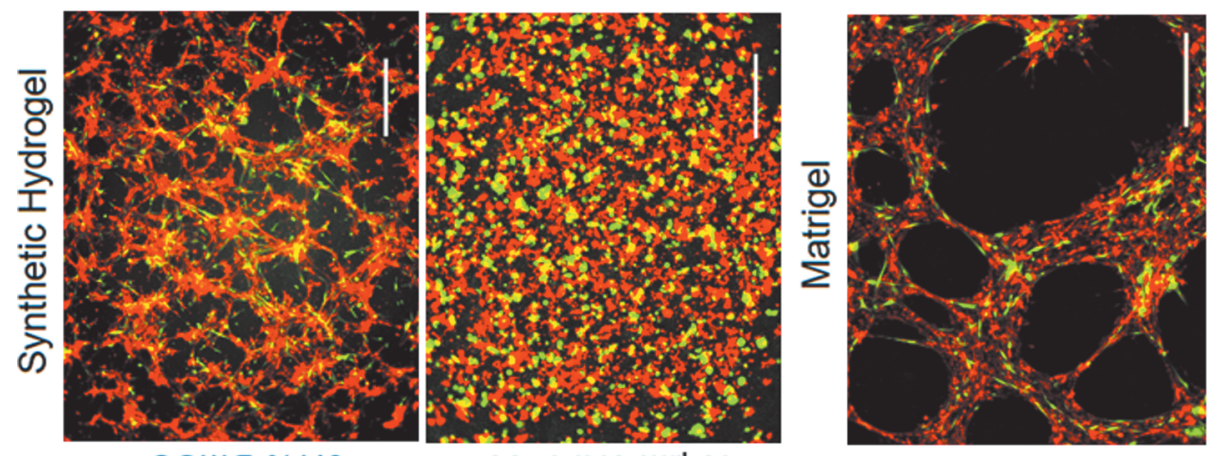

OSWa \%เ'0

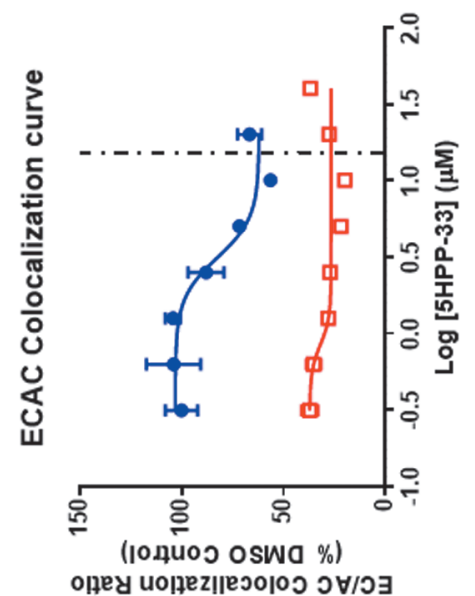

m OSWO \% 0

$\varepsilon \varepsilon-d d H G$ Wrl ot

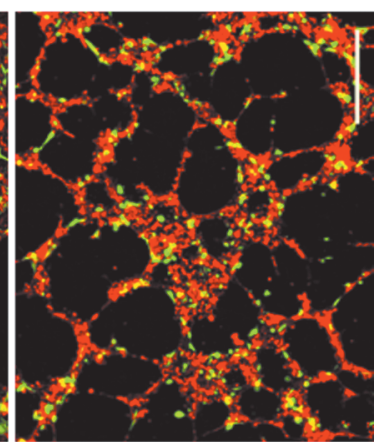

$\varepsilon \varepsilon-d d H G$ wrl $0 t$ 

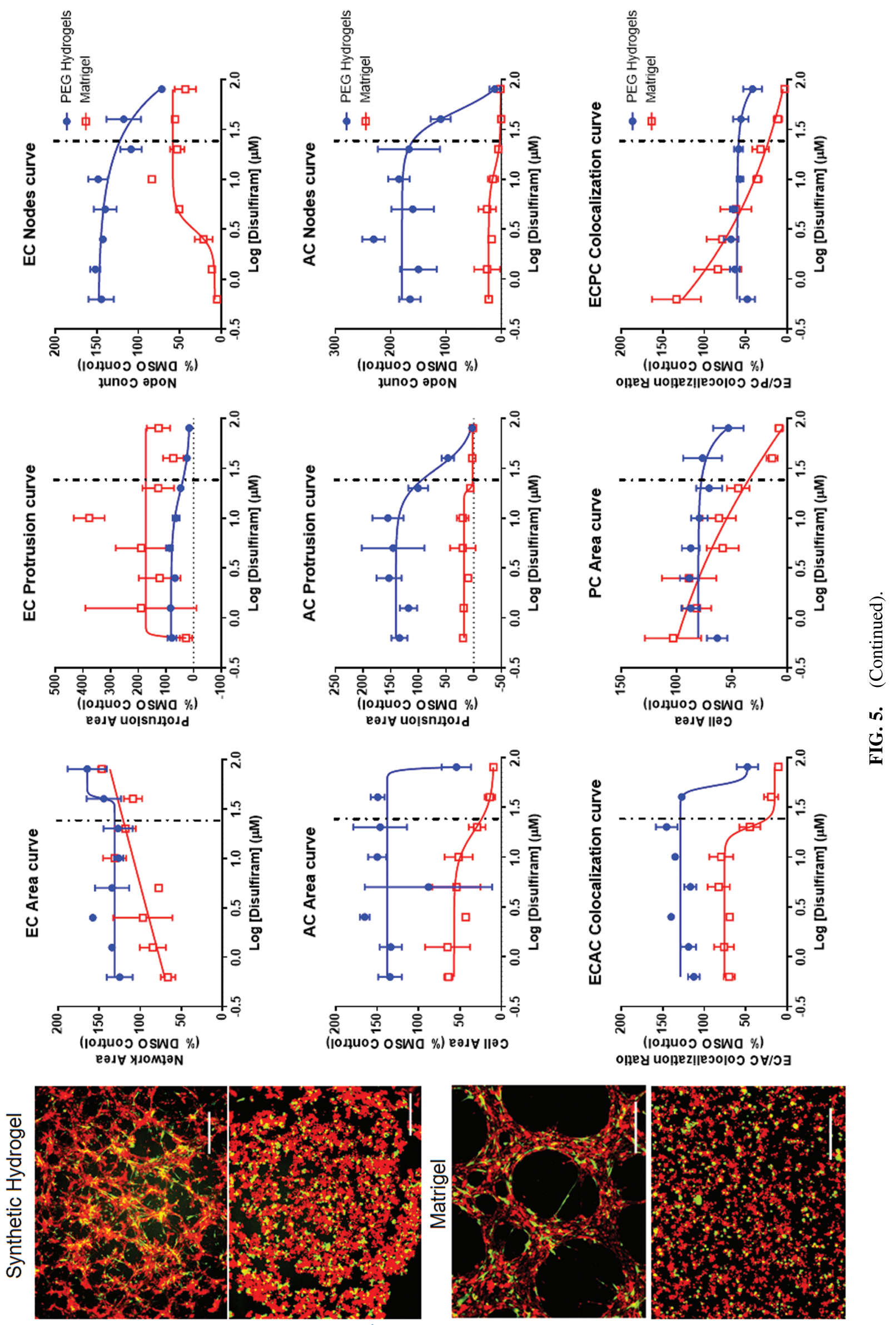

○ OSWO \% 0

med!!nns! Wrl 08

OSWO \% ${ }^{\circ} 0$

med!!j|ns! Wrl $_{08}$ 

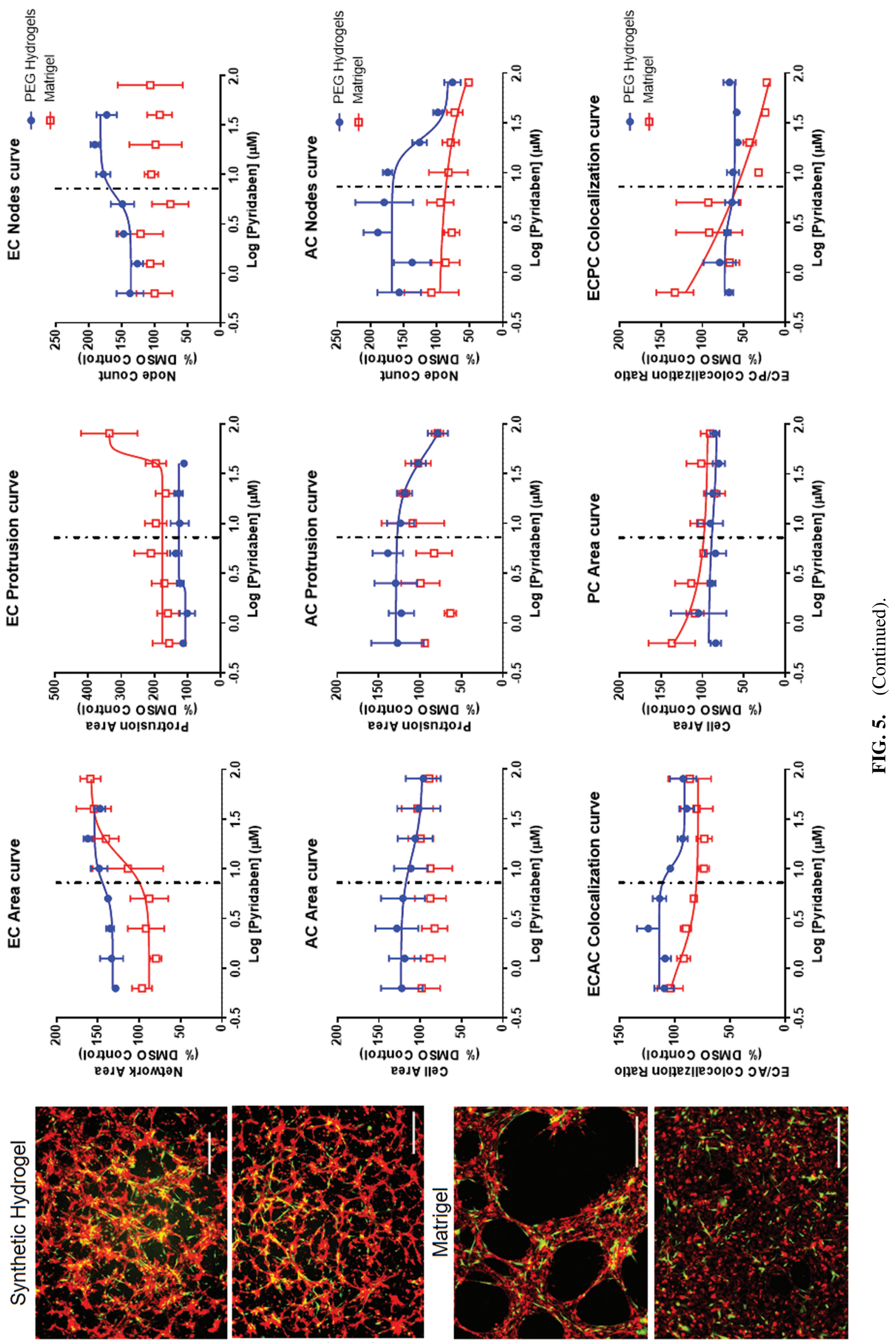

uəqep! $1 \Lambda_{d} \mathrm{Wrl}_{08}$

OSWa \% เ'0

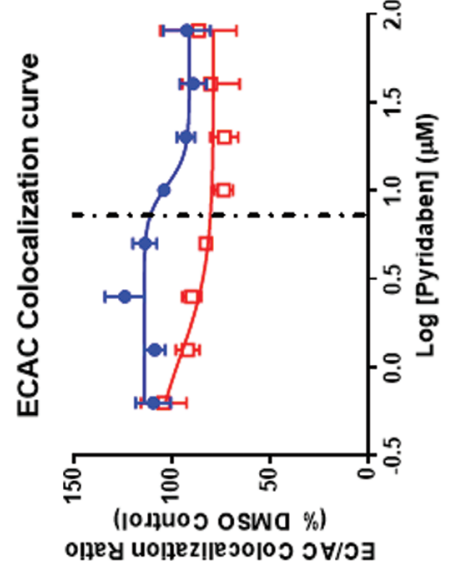

نே 


\section{Comparison of dose-response with NVU models plated on Matrigel}

When chemical dose-response was compared between Matrigel and PEG-based NVU models, fewer measurement methods generated usable $\mathrm{AC}_{50}$ values in the Matrigelbased system. Chemical treatments often caused the formation of fine cell networks rather than broad cell sheets on Matrigel (Fig. 5 and Supplementary Fig. S5), likely owing to the differential mechanisms of network formation observed on PEG hydrogels and Matrigel. ${ }^{33}$ Because of this, network area measurements were more effective in detecting $\mathrm{AC}_{50}$ values on Matrigel than on PEG hydrogels. EC protrusion measurements were often not suitable for generating $\mathrm{AC}_{50}$ values on Matrigel, as cells generally formed structures that lacked finer protrusions and bridges present in the PEGbased NVU models (Fig. 5, all representative dose-response curves in Supplementary Fig. S5). If any morphological metrics generated usable $\mathrm{AC}_{50}$ values in both Matrigel and PEGbased NVU models, Matrigel models generated higher $\mathrm{AC}_{50}$ values that had more variation than equivalent PEG-based NVU models. This signifies decreased sensitivity and consistency by the Matrigel-based NVU model.

Several chemicals were effective in detecting $\mathrm{AC}_{50}$ values on Matrigel and PEG-based NVU models. These include 5HPP33, fluazinam, and disulfiram, with 5HPP33 generating the most usable $\mathrm{AC}_{50}$ values in both models. Interestingly, pyridaben only generated $\mathrm{AC}_{50}$ values for endothelial network area. At high concentrations, pyridaben caused confluent cell sheet formation rather than network formation. In addition, this change in cell behavior did not impact $\mathrm{AC}$ or PC morphologically. Phenolphthalein and triclocarban lost much of their ability to generate $\mathrm{AC}_{50}$ values on the Matrigel-based assay. Notably, 1-hydroxypyrene lost all ability to generate converging sigmoidal curves in Matrigel-based models. One chemical that generated similar results in PEG and Matrigel-based assays was quercetin. It generated $\mathrm{AC}_{50}$ values largely from EC-based morphological changes in the NVU model on both PEG hydrogels and Matrigel. Finally, reserpine was not able to generate many usable $\mathrm{AC}_{50}$ values in either the Matrigel or PEG NVU model, and 4nonylphenol branched generated no usable $\mathrm{AC}_{50}$ values in both NVU models.

\section{Discussion}

In this study, we developed an NVU model to detect and characterize antiangiogenic activity by potential vascular disrupting chemicals. It consisted of iPSC-derived ECs and ACs, as well as human brain-derived PCs seeded onto a proangiogenic PEG hydrogel. The model detected 10 critical priority chemicals from a panel of $38 \mathrm{pVDCs}$ and characterized their effects on neurovascular angiogenesis. The combination of nine metrics of NVU morphology generated results that were uniquely associated with each of the tested chemicals. Identifying factors of each chemical included whether measurements generated usable $\mathrm{AC}_{50}$ values, effective dosing levels, and cell types that were sensitive to chemical exposure. Chemicals were dosed for a 24-hour incubation period to achieve rapid detection of vascular disruptive chemicals.

We observed that chemical exposure initiated a diversity of morphological changes in NVU models constructed on
PEG hydrogels. These changes included cells forming smaller disconnected structures rather than well-connected capillary networks, cells adopting rounded nonadhesive morphologies, and capillary networks meshing at varying densities. In addition, exposure with certain chemicals would preferentially change morphologies in certain cell types over others. The measurement methods employed here were selected to account for all of the mentioned possibilities in each of the cell types included in the NVU model.

One of the expectations associated with this work is that the combination of unique morphological changes imposed by the chemicals tested here and the dose dependence of detected changes will predict which cell signaling pathways were activated by uncharacterized chemicals. The PEG-based NVU model is a promising tool toward adverse outcome pathway prediction due to its ability to detect a wide variety of morphological changes during chemical exposure. Moreover, the effective chemical concentrations detected on PEG hydrogels corroborated with known concentrations found in the EPA ToxCast database $^{50}$ (see Screening Predicted Vascular Inhibitors from the ToxCast Library section). Therefore, we believe that the effective chemical concentrations detected by the PEG-based NVU mode are, in most cases, as predictive of physiological activity as more established models.

Although some chemicals matched the median $\mathrm{AC}_{50}$ of ToxCast vascular assays more closely than otherspyridaben, fluazinam, and quercetin were most accuratemost of $\mathrm{AC}_{50}$ values observed here still fell within the range of effective vascular ToxCast $\mathrm{AC}_{50}$ values (data not shown). The exceptions to this are 1-hydroxypyrene, reserpine, and 4-nonylphenol branched, which derived $\mathrm{AC}_{50}$ values far out of range of ToxCast's vascular $\mathrm{AC}_{50}$ values. Note that ToxCast vascular assays currently do not include data pertaining to EC/PC/AC cocultures, and much of the data were derived from cell-based assays, whereas less of the data were derived from functional tubulogenesis assays. We believe that the coculture conditions used here may have a role in changing the effective doses of these three chemicals. This also highlights the need to develop increasingly biologically relevant toxicity assays to improve the predictability of toxicity databases in more biological contexts.

Although the PEG-based NVU model was able to detect chemical activity on the NVU model, it was not known whether a gold-standard material such as Matrigel would be an appropriate material for use in a similar suite of analyses. In addition, we aimed to demonstrate that the chemically defined environment presented in the PEG-based NVU model would improve the sensitivity of the assay over the Matrigel-based NVU model. Our results here suggest that although Matrigel-based models were generally less effective in detecting chemical activity through the tested quantification methods, Matrigel-based models were as effective as PEG-based NVU models in detecting activity by certain chemicals, including 5HPP-33 and disulfiram. Had Matrigel not detected chemical activity by any of the chemicals tested in these studies, it would have suggested that our analysis methods were fundamentally inappropriate for use in Matrigel-based assays, and that a different suite of analysis methods would need to be developed. The fact that the analyses did not fail across all analyses under all chemical treatments at least showed that the analysis methods were appropriate on both PEG hydrogel and Matrigel-based assays. 
The main cause of undetected chemical activity on Matrigel was due to the fact that chemicals mostly modified the thickness of network structures between single-cell-wide strands to confluent cell sheets. They rarely prevented cell spreading and rarely changed the connectivity of endothelial networks. Therefore, we predict that the Matrigel-based NVU model would have a limited ability to differentiate between chemical modes of action. Owing to the generally poor sensitivity and reproducibility of Matrigel-based assays, ${ }^{33}$ further incorporation of Matrigel conditions in NVU chemical screens is not recommended. However, the use of Matrigel to elucidate further ECM signals for incorporation into PEG hydrogels is a promising application of the material. Refer to Supplementary Data for detailed discussion on possible chemical mechanisms of action detected using the coculture system.

Our vascular models cultured multiple neurovascular cell types in direct contact with one another and directed their formation into branched capillary-like networks. Specifically, our screening experiments explored how chemical activity affects neurovascular capillary network assembly. Our work explored chemically mediated changes the ability of NVU cells to assemble into vascular networks. Another group has generated branched capillary-like networks by coculturing ECs with neural stem cells on Matrigel substrates, ${ }^{52}$ potentially leading to the generation of a wide variety of cell types included in the NVU model beyond PCs and ACs. The defining features of our work were that we cocultured ECs with defined, differentiated populations of PCs and ACs, and performed experiments on chemically defined PEG hydrogels. We expect this to contribute to the generation of consistent tubulogenesis assays that may be performed over the course of 24 hours rather than 7 days.

We selected a coculture condition with the lowest density number of perivascular support cells that significantly supported capillary network formation above EC-only controls. This was an appropriate starting point to investigate how perivascular cells influence angiogenic responses to chemical exposure. Our coculture condition was also expected to simulate scenarios where the neurovasculature is particularly vulnerable to chemically mediated disruption. These scenarios include early neurovascular development, ${ }^{53,54}$ where perivascular support cells are present in low densities, and the decreased presence of PCs due to platelet-derived growth factor BB knockdown. ${ }^{55}$

With the varying ratios of ECs with perivascular cells that can be included in the NVU model, the application of the model is expandable to cover a variety of chemical exposure scenarios. The density of supporting cell types surrounding capillaries varies between regions of the CNS. ${ }^{56-58}$ For example, cortex vasculature has the greatest density of PCs in the CNS $\left(80 \%\right.$ coverage $\left.{ }^{56,59}\right)$, whereas spinal cord has the lowest density of PCs (48\%-68\% coverage $\left.{ }^{56,59}\right)$. In addition, the developing mouse spinal cord contains a greater amount of ACs than the developing brain (up to 97-fold higher ALDH1L1 abundance in spinal cord by Western blot to quantify ACs presence ${ }^{58}$ ). Differential densities of perivascular cells can result in differential adverse outcomes to chemical exposure in varying areas of the CNS and in varying stages of development. Future experiments will change endothelial and perivascular cell densities to model varying aspects of NVU pathology or development, and how those situations change NVU responses to chemical exposure.

\section{Conclusions}

An organotypic NVU model was developed to detect and characterize the disruptive effects of chemical exposure on human neurovascular tissue. The morphological changes to the NVU and its individual cellular components were unique to each chemical applied to the model. The use of PEG-based NVU models demonstrated greater sensitivity and consistency of chemical detection over equivalent Matrigel-based NVU models. There is a need to perform accurate risk assessments on large number of uncharacterized chemicals to guard against the potential harm they present to human health. In the context of neurovascular pathology, highthroughput screening and high-content analysis of neurovascular disruption may facilitate risk assessments of large chemical libraries by identifying critical chemical hazards, infer mechanisms of neurovascular disruption, and infer vulnerable cell types during an exposure event. We expect that this method will increase the speed and throughput of drug and chemical screening assays.

\section{Acknowledgments}

The authors acknowledge funding from the NIH (Grant numbers R01HL093282-01A1, R21EB016381-01, R01EB10039, R24 EY022883, R01 EY026078, and P30 EY016665), the National Science Foundation (Grant number GE-0718123), the EPA (STAR grant No. 83573701, the Chemical Safety for Sustainability Research Program, the Virtual Tissue Models Project, and the National Center for Computational Biology), the UW-Madison Molecular and Environmental Toxicity Center Training Program (Grant number NIH T32 ES007015), the Retina Research Foundation, and an unrestricted departmental award from Research to Prevent Blindness. The pVDC panel tested in this study was provided by the EPA. This study made use of the National Magnetic Resonance Facility at Madison, which is supported by NIH grant P41GM103399 (NIGMS), old number: P41RR002301. Equipment was purchased with funds from the University of Wisconsin-Madison, the NIH P41GM103399, S10RR02781, S10RR08438, S10RR023438, S10RR025062, and S10RR029220), the NSF (Grant numbers DMB-8415048, OIA-9977486, and BIR-9214394), and the USDA.

\section{Author Disclosure Statement}

W.L.M. is a founder and stockholder for StemPharm, Inc. All other authors have no competing financial interests.

\section{Supplementary Material}

Supplementary Data

Supplementary References

Supplementary Figure S1

Supplementary Figure S2

Supplementary Figure S3

Supplementary Figure S4

Supplementary Figure S5

Supplementary Figure S6

Supplementary Figure S7

Supplementary Data File S1

Supplementary Data File S2 


\section{References}

1. Hartung T, Rovida C. Chemical regulators have overreached. Nature 2009:460;1080-1081.

2. Mueller D, Krämer L, Hoffmann E, et al. 3D organotypic HepaRG cultures as in vitro model for acute and repeated dose toxicity studies. Toxicol In Vitro 2014:28;104-112.

3. LeCluyse EL, Witek RP, Andersen ME, et al. Organotypic liver culture models: Meeting current challenges in toxicity testing. Crit Rev Toxicol 2012:42;501-548.

4. Saili KS, Zurlinden TJ, Schwab AJ, et al. Blood-brain barrier development: Systems modeling and predictive toxicology. Birth Defects Res 2017:109;1680-1710.

5. Sweeney MD, Kisler K, Montagne A, et al. The role of brain vasculature in neurodegenerative disorders. Nat Neurosci 2018:21;1318-1331.

6. Zlokovic BV. Neurovascular pathways to neurodegeneration in Alzheimer's disease and other disorders. Nat Rev Neurosci 2011:12;723-738.

7. Roher AE, Debbins JP, Malek-Ahmadi M, et al. Cerebral blood flow in Alzheimer's disease. Vasc Health Risk Manag 2012:8;599-611.

8. Nelson AR, Sweeney MD, Sagare AP, et al. Neurovascular dysfunction and neurodegeneration in dementia and Alzheimer's disease. Biochim Biophys Acta 2016:1862;887900.

9. Derejko M, Sławek J, Wieczorek D, et al. Regional cerebral blood flow in Parkinson's disease as an indicator of cognitive impairment. Nucl Med Commun 2006:27;945-951.

10. Firbank MJ, Colloby SJ, Burn DJ, et al. Regional cerebral blood flow in Parkinson's disease with and without dementia. Neuroimage 2003:20;1309-1319.

11. Ichim TE, Solano F, Glenn E, et al. Stem cell therapy for autism. J Transl Med 2007:5;30.

12. Bjørklund G, Kern JK, Urbina MA, et al. Cerebral hypoperfusion in autism spectrum disorder. Acta Neurobiol Exp (Wars) 2018:78;21-29.

13. Ryan CM. Diabetes and brain damage: More (or less) than meets the eye? Diabetologia 2006:49;2229-2233.

14. Moss HE. Retinal vascular changes are a marker for cerebral vascular diseases. Curr Neurol Neurosci Rep 2015:15;40.

15. Nielsen RB, Egefjord L, Angleys H, et al. Capillary dysfunction is associated with symptom severity and neurodegeneration in Alzheimer's disease. Alzheimers Dement 2017:13;1143-1153.

16. Di Marco LY, Venneri A, Farkas E, et al. Vascular dysfunction in the pathogenesis of Alzheimer's disease-A review of endothelium-mediated mechanisms and ensuing vicious circles. Neurobiol Dis 2015:82;593-606.

17. Bonkowski D, Katyshev V, Balabanov RD, et al. The CNS microvascular pericyte: Pericyte-astrocyte crosstalk in the regulation of tissue survival. Fluids Barriers CNS 2011:8;8.

18. Al Ahmad A, Taboada CB, Gassmann M, et al. Astrocytes and pericytes differentially modulate blood-brain barrier characteristics during development and hypoxic insult. J Cereb Blood Flow Metab 2011:31;693-705.

19. McCarthy RC, Kosman DJ. Mechanisms and regulation of iron trafficking across the capillary endothelial cells of the blood-brain barrier. Front Mol Neurosci 2015:8;31.

20. Abbott NJ, Rönnbäck L, Hansson E. Astrocyte-endothelial interactions at the blood-brain barrier. Nat Rev Neurosci 2006:7;41-53.

21. Ribatti D. Judah Folkman, a pioneer in the study of angiogenesis. Angiogenesis 2008:11;3-10.
22. Hughes C, Postovit L, Lajoie G. Matrigel: A complex protein mixture required for optimal growth of cell culture. Proteomics 2010:10;1886-1890.

23. Smithmyer ME, Sawicki LA, Kloxin AM. Hydrogel scaffolds as in vitro models to study fibroblast activation in wound healing and disease. Biomater Sci 2014:2;634-650.

24. Cushing MC, Anseth KS. Materials science. Hydrogel cell cultures. Science 2007:316;1133-1134.

25. Folkman J, Haudenschild C. Angiogenesis in vitro. Nature 1980:288;551-556.

26. Lutolf MP, Hubbell JA. Synthetic biomaterials as instructive extracellular microenvironments for morphogenesis in tissue engineering. Nat Biotechnol 2005:23;47-55.

27. Murphy WL, McDevitt TC, Engler AJ. Materials as stem cell regulators. Nat Mater 2014:13;547-557.

28. Murrow LM, Weber RJ, Gartner ZJ. Dissecting the stem cell niche with organoid models: An engineering-based approach. Development 2017:144;998-1007.

29. Cruz-Acuna R, Garcia AJ. Synthetic hydrogels mimicking basement membrane matrices to promote cell-matrix interactions. Matrix Biol 2017:57-58;324-333.

30. Nguyen EH, Zanotelli MR, Schwartz MP, et al. Differential effects of cell adhesion, modulus and VEGFR-2 inhibition on capillary network formation in synthetic hydrogel arrays. Biomaterials 2014:35;2149-2161.

31. Zanotelli MR, Ardalani H, Zhang J, et al. Stable engineered vascular networks from human induced pluripotent stem cell-derived endothelial cells cultured in synthetic hydrogels. Acta Biomater 2016:35;32-41.

32. Belair DG, Schwartz MP, Knudsen T, et al. Human iPSCderived endothelial cell sprouting assay in synthetic hydrogel arrays. Acta Biomater 2016:39;12-24.

33. Nguyen E, Daly W, Le NN, et al. Versatile synthetic alternatives to Matrigel for vascular toxicity screening and stem cell expansion. Nat Biomed Eng 2017:2017;0096.

34. Schwartz MP, Hou Z, Propson NE, et al. Human pluripotent stem cell-derived neural constructs for predicting neural toxicity. Proc Natl Acad Sci U S A 2015:112; 12516-12521.

35. Barry C, Schmitz MT, Propson NE, et al. Uniform neural tissue models produced on synthetic hydrogels using standard culture techniques. Exp Biol Med (Maywood) 2017: 242;1679-1689.

36. Chan XY, Black R, Dickerman K, et al. Three-dimensional vascular network assembly from diabetic patient-derived induced pluripotent stem cells. Arterioscler Thromb Vasc Biol 2015:35;2677-2685.

37. Nolan DJ, Ginsberg M, Israely E, et al. Molecular signatures of tissue-specific microvascular endothelial cell heterogeneity in organ maintenance and regeneration. Dev Cell 2013: 26;204-219.

38. Lippmann ES, Al-Ahmad A, Azarin SM, et al. A retinoic acid-enhanced, multicellular human blood-brain barrier model derived from stem cell sources. Sci Rep 2014:4;4160.

39. Lippmann ES, Azarin SM, Kay JE, et al. Derivation of blood-brain barrier endothelial cells from human pluripotent stem cells. Nat Biotechnol 2012:30;783-791.

40. Katt ME, Xu ZS, Gerecht S, et al. Human brain microvascular endothelial cells derived from the BC1 iPS cell line exhibit a blood-brain barrier phenotype. PLoS One 2016:11; e0152105.

41. Minami H, Tashiro K, Okada A, et al. Generation of brain microvascular endothelial-like cells from human induced pluripotent stem cells by co-culture with C6 glioma cells. PLoS One 2015:10;e0128890. 
42. Yuan F, Fang KH, Cao SY, et al. Efficient generation of region-specific forebrain neurons from human pluripotent stem cells under highly defined condition. Sci Rep 2015:5; 18550.

43. Shaltouki A, Peng J, Liu Q, et al. Efficient generation of astrocytes from human pluripotent stem cells in defined conditions. Stem Cells 2013:31;941-952.

44. Chandrasekaran A, Avci HX, Leist M, et al. Astrocyte differentiation of human pluripotent stem cells: New tools for neurological disorder research. Front Cell Neurosci 2016:10;215.

45. Xu J, Gong T, Heng BC, et al. A systematic review: Differentiation of stem cells into functional pericytes. FASEB J 2017:31;1775-1786.

46. Iwata Y, Klaren WD, Lebakken CS, et al. High-content assay multiplexing for vascular toxicity screening in induced pluripotent stem cell-derived endothelial cells and human umbilical vein endothelial cells. Assay Drug Dev Technol 2017:15;267-279.

47. Knudsen TB, Kleinstreuer NC. Disruption of embryonic vascular development in predictive toxicology. Birth Defects Res C Embryo Today 2011:93;312-323.

48. Kleinstreuer NC, Judson RS, Reif DM, et al. Environmental impact on vascular development predicted by high-throughput screening. Environ Health Perspect 2011:119;1596-1603.

49. El-Masri H, Kleinstreuer N, Hines RN, et al. Integration of life-stage physiologically based pharmacokinetic models with adverse outcome pathways and environmental exposure models to screen for environmental hazards. Toxicol Sci 2016:152;230-243.

50. USEPA. ToxCast \& Tox 21 Summary Files from ToxCast Dashboard.

51. Franco M, Roswall P, Cortez E, et al. Pericytes promote endothelial cell survival through induction of autocrine VEGF-A signaling and Bcl-w expression. Blood 2011: 118;2906-2917.

52. Chou CH, Sinden JD, Couraud PO, et al. In vitro modeling of the neurovascular environment by coculturing adult human brain endothelial cells with human neural stem cells. PLoS One 2014:9; e106346.

53. Moretti R, Pansiot J, Bettati D, et al. Blood-brain barrier dysfunction in disorders of the developing brain. Front Neurosci 2015:9;40.

54. Saunders NR, Liddelow SA, Dziegielewska KM. Barrier mechanisms in the developing brain. Front Pharmacol 2012: $3 ; 46$.

55. Nikolakopoulou AM, Zhao Z, Montagne A, et al. Regional early and progressive loss of brain pericytes but not vascular smooth muscle cells in adult mice with disrupted plateletderived growth factor receptor- $\beta$ signaling. PLoS One 2017:12;e0176225.

56. Wilhelm I, Nyúl-Tóth Á, Suciu M, et al. Heterogeneity of the blood-brain barrier. Tissue Barriers 2016:4;e1143544.

57. Villaseñor R, Kuennecke B, Ozmen L, et al. Region-specific permeability of the blood-brain barrier upon pericyte loss. J Cereb Blood Flow Metab 2017:37;3683-3694.

58. Yoon H, Walters G, Paulsen AR, et al. Astrocyte heterogeneity across the brain and spinal cord occurs developmentally, in adulthood and in response to demyelination. PLoS One 2017:12; 0180697.

59. Winkler EA, Sengillo JD, Bell RD, et al. Blood-spinal cord barrier pericyte reductions contribute to increased capillary permeability. J Cereb Blood Flow Metab 2012:32;18411852.

Address correspondence to: Dr. Nader Sheibani

Department of Ophthalmology and Visual Sciences University of Wisconsin School of Medicine and Public Health 1111 Highland Avenue, 9453 WIMR Madison, WI 53705

E-mail: nsehaibanikar@wisc.edu 